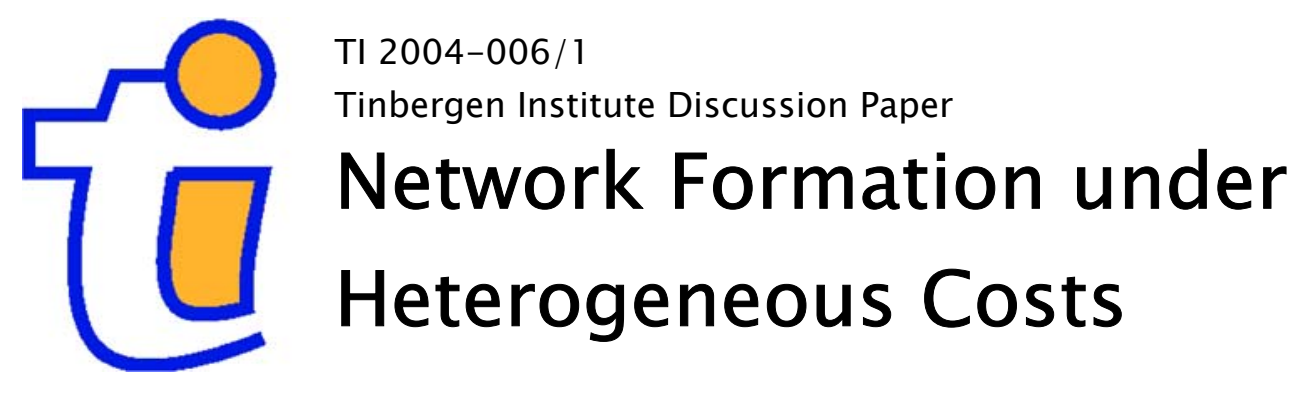

Jurjen Kamphorst

Gerard van der Laan

Department of Econometrics, Vrije Universiteit Amsterdam, and Tinbergen Institute. 


\section{Tinbergen Institute}

The Tinbergen Institute is the institute for economic research of the Erasmus Universiteit Rotterdam, Universiteit van Amsterdam, and Vrije Universiteit Amsterdam.

Tinbergen Institute Amsterdam

Roetersstraat 31

1018 WB Amsterdam

The Netherlands

Tel.: $\quad+31(0) 205513500$

Fax: $\quad+31(0) 205513555$

Tinbergen Institute Rotterdam

Burg. Oudlaan 50

3062 PA Rotterdam

The Netherlands

Tel.: $\quad+31(0) 104088900$

Fax: $\quad+31(0) 104089031$

Please send questions and/or remarks of nonscientific nature to driessen@tinbergen.nl.

Most TI discussion papers can be downloaded at http://www.tinbergen.nl. 


\title{
Network Formation Under Heterogeneous Costs: The Multiple Group Model ${ }^{1}$
}

\author{
Jurjen Kamphorst ${ }^{2}$ and Gerard van der $\operatorname{Laan}^{3}$
}

\begin{abstract}
It is widely recognized that the shape of networks influences both individual and aggregate behavior. This raises the question which types of networks are likely to arise. Our paper fits into the literature that addresses this question.

The paper continues on the work of Galeotti, Goyal and Kamphorst (2003). In their paper they investigate a model of network formation, where players are divided into groups. The costs of a link between any pair of players are increasing in the distance between the groups that these players belong to. They apply the solution concept of minimal curb sets to the case of two groups.

In this paper we give a full characterization of the networks induced by a minimal curb set for any number of groups. To do so, we show that in our multiple group model each minimal curb set is a so-called super-tight curb set, that is a minimal curb set satisfying the condition that in each state of the set every player has the same best reply. From the proof it follows that every recurrent class of an unperturbed best reply dynamics is a minimal (super-tight) curb set and reversely. From this result we obtain the characterization of the types of networks that may arise in a minimal curb set. We show that such networks may have features that can not occur in networks with at most two groups. Nevertheless, local centrality and center-sponsorship are still important characteristics of the networks in minimal curb sets.
\end{abstract}

\footnotetext{
${ }^{1}$ This research is part of the research program "Strategic and Cooperative Decision Making". We thank Andrea Galeotti, Sanjeev Goyal and Vitaly Pruzhansky.

${ }^{2}$ Department of Econometrics and Tinbergen Institute, Free University, De Boelelaan 1105, Amsterdam, The Netherlands. E-mail: jkamphorst@econ.vu.nl

${ }^{3}$ Department of Econometrics and Tinbergen Institute, Free University, De Boelelaan 1105, Amsterdam, The Netherlands. E-mail: glaan@econ.vu.nl
} 


\section{INTRODUCTION}

Imagine a world with individuals that would benefit from trade with each other. Yet the world is divided into several cultures and individuals from cultures more alien to each other find it more difficult to interact than individuals from cultures more similar to each other. In our world, we have seen that some people, often merchants, have invested into making contact with people from other cultures. And through those contacts information and goods were exchanged between individuals of those different cultures. In this paper we examine a world in which the agents are gathered into groups, according to their characteristics ${ }^{4}$. In this world, agents exchange valuable information with everyone that they have contact with, including the information that they received from others. However, before two agents have contact, one of the agents has to make the contact. Naturally, the more similar two players are, the easier contact will be made.

This paper fits into the quickly expanding literature on the formation of social and economic networks. The structure of networks can significantly affect individual and aggregate behavior ${ }^{5}$. From that perspective it is worthwhile to investigate which networks are likely to arise in different circumstances. Many papers have been published on this subject ${ }^{6}$.

In this paper we generalize and extend results on network formation obtained by Bala and Goyal (2000) and Galeotti, Goyal and Kamphorst (2003), to be referred to as BG and GGK respectively. In BG a set of homogeneous agents is considered. Because the network formation will be analyzed within a game-theoretic framework, in the sequel the agents are called players. Two players are said to be linked to each other when one of the players makes contact with the other player. BG assume that the cost of a link between two players is completely paid by the player who takes the initiative to make the contact. Therefore the contacting player is said to be the sponsor: he sponsors (pays for) the link between the two players. It is assumed that each link is equally costly. Two players are connected to each other when there is at least one path of links between them, i.e. two players $i$ and $j$ are connected if there is a sequence of players $i_{1}, i_{2}, \ldots, i_{j}$ such that $i=i_{1}$, $j=i_{k}$ and $i_{j}$ is linked to $i_{j+1}, j=1,2, \ldots, k-1$. Players benefit from being connected with other players. BG assume that the benefits are independent

\footnotetext{
${ }^{4}$ For example, according to the organisation, country, culture, social class or geographical area that they belong to. Another possibility is for instance to group scientists according to their field of specialization.

${ }^{5}$ See for instance Burt (1992), Montgomery (1991), Granovetter (1974), Coleman (1996), Barabási (2002) and Watts (2003).

${ }^{6}$ For network formation models with homogeneous agents see for instance Dutta and Jackson (2000), Falk and Kosfeld (2003), Goyal (1993), Haller and Sarangi (2001), Jackson and Wolinski (1996), and Watts $(2001,2002)$. Network formation models with heterogenous agents have been studied by e.g. Johnson and Gilles (2000) and McBride (2002). Jackson (2003) is an overview paper of the network formation literature that uses cooperative game theory (this is at the moment of writing downloadable from his website).
} 
from the sponsoring players and the length of the path(s) between two connected players. So, while the costs of a link are one-sided, the benefits are two-sided: when one player sponsors a link to another player, both obtain the same benefit. Moreover, there is no decay or path dependency, i.e. the benefit from being connected to another player is independent of the length of the path(s) and the number of different paths between the two players. Finally, any connection gives the same benefit. So, the total benefit to a player is equal to some constant times the number of players with which he is connected. From this it follows that the payoff to a player is equal $K b-L c$, where $b$ is the benefit per connection, $K$ the number players to which he is connected, $c$ the costs of a link and $L$ the number of links sponsored by the player. Observe that both costs and values (benefits) are homogeneous.

For instance, consider a three player network with one link between Pete and John, sponsored by John. Then the payoff to Pete is $b-c$, the payoff to John is $b$ and the payoff to the third player Sarah is 0. Although also John benefits from the link that Pete sponsors, only Pete incurs the costs of that link. For example, when Pete makes a phone call to John, only Pete pays for the call, while both Pete and John may benefit from the exchange of information. When also Sarah is sponsoring a link to John, then Pete is connected to her through his link to John and her link to John. The payoffs are now $2 b-c$ for both Pete and Sarah and $2 b$ for John. When in addition to the links above Sarah also sponsors a link to Pete, then the payoff to Pete and John does not change: they still incur the same costs, and neither of them receives more benefits, because neither the length of the connection to Sarah nor the number of different paths to other players matter. However, the additional link decreases Sarah's payoff to $2 b-2 c$, because she now also incurs the costs of the additional link.

Within this framework a network is called a (strict) Nash network if the network is a (strict) Nash equilibrium, i.e. if every player plays a (strict) best reply to this network. A strategy is a strict best reply versus a network, if it is the unique best reply against that network. A weak Nash network is a non-strict Nash network. BG show that if the costs of a link are higher than the benefit per connection, the only strict Nash network is empty (no links at all). When the costs of a link are lower than the benefit per connection, then each strict Nash network is non-empty (has at least one link). Moreover, any strict Nash network is connected (each player is connected to any other player) and is a so-called center-sponsored star, i.e. there is precisely one player sponsoring links and this player sponsors a link to any other player. BG also show that in a dynamic setting where players may revise their strategies as if they are myopically rational, the dynamics always converges to a strict Nash network. An example of a center-sponsored star of 4 players is depicted in Figure 1. Player 1 is there the central player, who sponsors a link to each other player. 


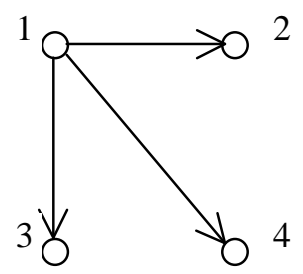

Figure 1. Center-Sponsored Star

These results are generalized in GGK (Galeotti, Goyal and Kamphorst, 2003) by relaxing the assumptions of cost homogeneity and value homogeneity. GGK find that if either costs of links or the benefit per connection are heterogeneous, a non-empty strict Nash network may fail to be connected. So, under heterogeneity of costs or benefits there may exist strict Nash networks in which two players are not connected to each other. In particular: if costs are homogeneous and values are heterogeneous, then strict Nash networks may exist consisting of several center-sponsored star components (a subset of players is a component if it is connected and the subset becomes unconnected when adding any other player). If costs are heterogeneous, then any minimal (unconnected) network may arise as a strict Nash network (a network is minimal if the number of components increases when deleting any one link). Hence GGK conclude that both homogeneity in costs and in values are important determinants for the existence of connected strict Nash networks. Moreover, they conclude that cost heterogeneity is crucial for the shape (or architecture) of the networks that may arise as strict Nash networks, as without restrictions on the cost function any network with a minimal architecture may arise as a strict Nash network.

Since value heterogeneity is relatively unimportant for the architecture of the network, GGK proceed by assuming homogeneous value functions. Moreover, they restrict the heterogeneity of costs to be of a specific form. To do so, they assume that, according to certain characteristics, the players can be divided into $m$ groups, numbered from 1 to $m$, each group containing at least two players. It is further assumed that the numbering reflects the differences in the characteristics of a group. In particular, for simplicity it is assumed that the differences between two groups $h$ and $j$, can be measured by the distance between the groups being the absolute value $|h-j|$ of the difference between the numbers of the groups. For each player, the costs of a link to some other player is given by a cost function that is (weakly) increasing in the distance between the groups to which both players belong. Each player is assumed to have the same cost function $f$, with $f(k)$ the costs of a link between two players at distance $k, k=0, \ldots, m-1$. GGK call a link within a group an internal link and a link from a player to a player outside his own group an external link. Correspondingly they named this model the Insider-Outsider model. Since the characteristic feature of the model is the partitioning of the population into multiple groups, where there are many 

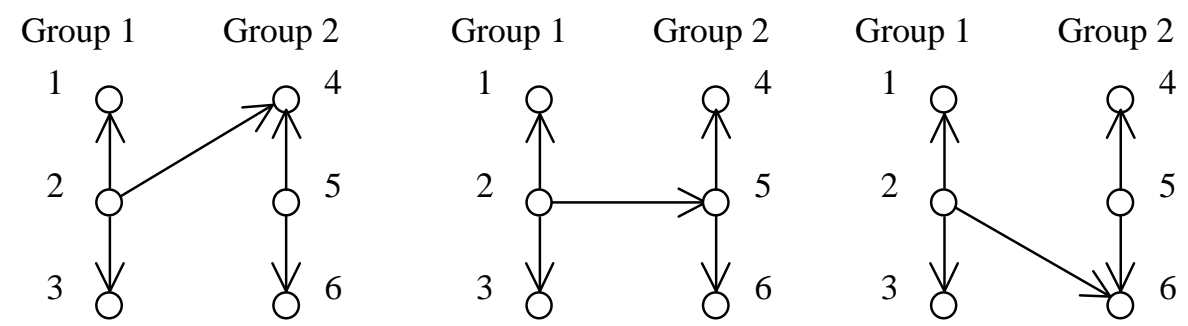

FiguRE 2. Three connected center-sponsored star with two groups of three players each.

different types of outsiders (depending on distance) from the point of view of any player, in this paper we prefer to call this model the Multiple Group model (MG model), and to refer to this as the Insider-Outsider model in the specific case of two groups.

For the MG model, GGK show that for high enough values of the costs $f(0)$ of internal links, the empty network is the unique strict Nash network. For low enough values of internal links and high enough values of external links of length one, any strict Nash network is a network in which each group forms a center-sponsored star component. If both the costs $f(0)$ of internal links and $f(1)$ of external links of length one are low enough, then any strict Nash network is a so-called generalized center-sponsored star. Such a network is minimally connected (i.e. deleting a link makes the network unconnected) and has a central player $i$ with the property that if we move along a path with subsequently the players $i_{1}, i_{2}, \ldots, i_{k}$ with $i_{1}=i$, then the link from $i_{j}$ to $i_{j+1}$ is sponsored by $i_{j}, j=1, \ldots, k-1$. Moreover, along each such a path, there is at most one player from each group, ignoring player $i$ himself. So, the group to which the central player $i$ belongs constitutes a center-sponsored star (i.e. $i$ sponsors a link to any other player in his group) and all other groups are completely fragmented (i.e. any path between two members of such a group contains a member of another group). Finally, GGK find that for low values of $f(0)$ and moderate values of $f(1)$ a strict Nash network does not exist.

For the case of just two groups, GGK subsequently characterize the networks in the recurrent classes of the dynamics with myopically rational players as specified by BG. It is shown that the dynamics converges to a recurrent class that may contain multiple Nash networks. If the recurrent class is nonsingleton ${ }^{7}$ then each network of the curb set is a weak Nash network in which both groups form a center-sponsored star and in which there is one single player, say $i$ (the same in each of these networks), that sponsors a link to one of the players in the other group. We call such a network a connected center-sponsored star network. Consider for example the three networks in Figure 2. These three networks are a recurrent class of the dynamics for

\footnotetext{
${ }^{7} \mathrm{~A}$ singleton recurrent class in these dynamics consists by definition of a strict Nash equilibrium.
} 
$f(0)<1, f(0)<f(1)<3$. For the same parameters, they are also Nash networks. But they are not strict Nash networks, because player 2 doesn't care to which player of group 2 he sponsors his link. Moreover, none of the other player cares which of the three alternatives player 2 chooses. Therefore player 2 may change this link from one period to the next. However, all other links are the same in each of these networks.

In the basic model with homogeneous costs and benefits of BG, each minimal curb set is a singleton strict Nash networks, justifying their focus on strict Nash networks from a dynamic perspective. However, in the MG model with heterogeneous costs, strict Nash networks do not exist for moderate values of $f(1)$ together with low values of $f(0)$. Moreover, strict Nash networks are not the only networks that may be part of a recurrent class. This raises the question whether focussing on the solution concept of strict Nash networks is too restrictive.

The obvious alternative, to consider all Nash networks, is also unattractive. It is a common feature of network formation models, including this one, that the concept of Nash equilibrium has very little bite. For instance, in the case of one group, for a range of costs, every minimal connected network is a Nash network. Moreover, in the dynamic setting that BG studied, all recurrent classes contain only one network, namely a strict Nash network. No weak Nash network survives such dynamics. And although some weak Nash networks survive the dynamics when there are two groups, in general most weak Nash networks can not survive. Thus the concept of all Nash networks appears too wide.

GGK therefore introduce the concept of minimal curb sets ${ }^{8}$. This static solution concept has considerable bite (excludes most networks) and nonempty solutions for all positive increasing cost functions. Moreover, in this model each minimal curb set is identical to one of the recurrent classes and vice versa. Thus a network is part of a minimal curb set if and only if it is part of a recurrent class of the best reply dynamics.

GGK also show that, if there are just two groups and the cost function is strictly increasing, a connected center-sponsored star is efficient, while the generalized center-sponsored star is not.

In this paper we generalize the analysis of GGK by allowing for an arbitrary number of groups. This generalization comes at a small cost, namely that we assume that the costs of a link are strictly increasing in its length. We show that in this model any network in a minimal curb sets is either a generalized center-sponsored star, or a connected center-sponsored star, or has an architecture given by a hybrid combination of these two types. On the other hand, many hybrid architectures can't arise in minimal curb sets. Therefore generalizing the two-group model to an $m$-group model is not straightforward. In fact, it appears to be extremely difficult, if not impossible, to give a characterization of architectures that may arise in a

\footnotetext{
${ }^{8}$ This notion was first introduced by Basu and Weibull (1991). See also section 3 of this paper.
} 
minimal curb set, since there is no a priori reason to exclude any network from the analysis. Fortunately, for the two-group case, GGK has shown that any minimal curb sets is best reply invariant, i.e. for any player holds that his best reply set is independent of the network in the curb set. Thus, when in such a network one player changes his collection of sponsored links to another collection set of links in his best reply set, the set of best replies of any other player does not change. So, the set of best replies of a player also remains unchanged even if all other players get a chance to choose another optimal collection of sponsored links. GGK call a minimal curb set that is best-reply invariant a super tight curb set. Since most networks cannot be part of a super tight curb set, the task of providing a full characterization of a super tight curb set is relatively light.

This sets the program for the research in this paper. We first prove that also in the multiple group case every minimal curb set is super tight. We do this by proving that every recurrent class of the best reply dynamics contains a super tight curb set. Because any minimal curb set contains such a recurrent class, any minimal curb set is super tight. Subsequently we are able to characterize the networks that can arise in minimal curb sets. Since many minimal curb sets feature a set of hybrid networks, generally the architectures are not efficient, although they are typically more efficient than a strict Nash network. Moreover, there are super tight curb sets of which the networks are connected center-sponsored stars. The networks of some of these minimal curb sets are efficient. Finally, we show which types of networks can arise if there are three groups.

Common results in the network formation literature such as (local) centrality and center-sponsorship also hold for the MG model.

The paper is organized as follows. Section 2 presents the model and the dynamics. Section 3 introduces minimal curb sets and super tight curb sets. Section 4 contains the main result that in the multiple group model all recurrent classes of the best reply dynamic are super tight curb sets, and therefore that all minimal curb sets are super tight. Section 5 then provides the characterization of the networks in super tight curb sets. Section 6 presents the types of networks in minimal curb sets when there are three groups, and section 7 concludes. Most of the proofs are relegated to the Appendices.

\section{The Model}

In this section we present the game-theoretic model of network formation under costs heterogeneity. We start by defining the stage game, i.e. the set of players, the strategy sets and the payoff functions. We then continue by stating the concept of a Nash network and the dynamics of the model. We finish this section with some additional terminology and notation that we need in the sequel of the paper. 
2.1. The stage game. We consider a set $N=\{1, \ldots, n\}$ of $n$ players, which would benefit from having contact with each other, for instance because they can share private information. A player can contact another player by establishing a link to that other player. The costs of a link is fully paid by the player that makes the contact, to be called the sponsor of a link. However, the benefits of the contact flow both ways: the sponsor receives all information that the recipient has, and the recipient receives all information that the sponsor has. So, in the model we have asymmetric costs and symmetric benefits: only the sponsor pays for the link, but both players benefit from a link.

Based on some well-specified characteristics the players are divided into $m$ groups, indexed by $\ell=1, \ldots, m$. We assume that, according to their characteristics, the groups can be seen to be located along a linear city, from group 1 on the left side to group $m$ on the right side. Hence, the absolute value of the difference in the numbers of two groups can be seen as a measure for the heterogeneity in the characteristics between them. With $N_{\ell} \subset N$ being the set of players in group $\ell$, the collection of subsets $N_{1}, \ldots, N_{m}$ forms a partition of $N$. It is assumed that $m \geq 2$ and $\left|N_{\ell}\right| \geq 2$ for all $\ell=1, \ldots, m$. We denote the collection of these groups by $\mathcal{N}=\left\{N_{1}, \ldots, N_{m}\right\}$.

To give the strategy sets of the link formation game, we denote a link between player $i$ and player $j$ sponsored by $i$, shortly a link from $i$ to $j$, by the ordered pair $(i, j)$. The set of all possible links sponsored by player $i$, $i \in N$, denoted by $\mathcal{L}_{i}$, is given by

$$
\mathcal{L}_{i}=\{(h, j) \in N \times N \mid h=i, j \neq i\} .
$$

The set of all feasible links is thus given by $\mathcal{L}=\cup_{i \in N} \mathcal{L}_{i}$. Each player (only) decides upon which links he actually sponsors. So, a strategy of player $i$ is a subset $g_{i}$ of $\mathcal{L}_{i}$ and the strategy space $\mathcal{G}_{i}$ of player $i$ is the collection of all subsets of $\mathcal{L}_{i}$, thus ${ }^{9} 10$

$$
\mathcal{G}_{i}=\left\{g_{i} \mid g_{i} \subseteq \mathcal{L}_{i}\right\}
$$

With some abuse of the standard game-theoretical notation, we define the strategy space $\mathcal{G}$ of subsets of $\mathcal{L}$ by ${ }^{11}$

$$
\mathcal{G}=\{g \mid g \subseteq \mathcal{L}\} .
$$

Clearly, any $g \in \mathcal{G}$ induces for any $i \in N$ a strategy $g_{i} \in \mathcal{G}_{i}$ given by

$$
g_{i}=\{(h, j) \in g \mid h=i\} .
$$

\footnotetext{
${ }^{9}$ Throughout the paper we will denote a subset by $\subseteq$ and a strict subset by $\subset$.

${ }^{10}$ Our notation of a strategy $g_{i}$ differs from the usual notation in the network formation literature, including GGK. We chose to denote the strategies of players by the set of links that they sponsor, whereas in the main literature the strategy of a player is denoted by an $n$-vector, say $h_{i}$. Then a strategy $g_{i} \in \mathcal{G}_{i}$ being a set of links corresponds to the $n$-vector $h_{i}$ with components $h_{i j}=1$ if $(i, j) \in g_{i}$ and $h_{i j}=0$ if $(i, j) \notin g_{i}$. We made this change because the usual notation becomes very cumbersome in our proofs.

${ }^{11}$ According to the formal approach in game theory, the strategy space is given by $\Pi_{i \in N} \mathcal{G}_{i}$ instead of $\mathcal{G}$.
} 


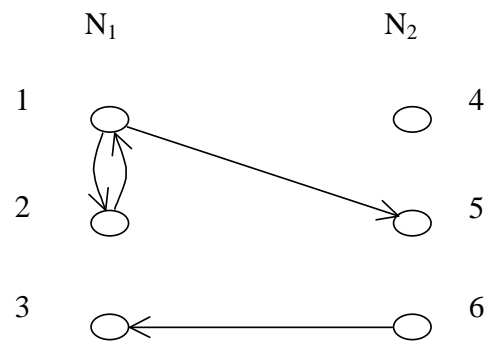

Figure 3. $g=\{(1,2),(1,5),(2,1),(6,3)\}$

Reversely, when player $i$ decides on strategy $g_{i} \in \mathcal{G}_{i}, i \in N$, then $g=$ $\cup_{i \in N} g_{i} \in \mathcal{G}$. So, without any confusion, any $g \in \mathcal{G}$ represents a strategy profile $g_{i}, i \in N$.

It is useful to observe that a strategy profile $g \in \mathcal{G}$ can be represented by a network, being a directed graph with the set of players as nodes and a directed edge between two nodes $i$ and $j$ if and only if $(i, j) \in g$. In the sequel we therefore often refer to a strategy profile as a network. Figure 3 is an example of a network in a game with $n=6, m=2, N_{1}=\{1,2,3\}$ and $N_{2}=\{4,5,6\}$, namely the network induced by the strategy profile $g=\{(1,2),(1,5),(2,1),(6,3)\}$. In all figures in this paper, we put players of the same group vertically to each other. So, every column of nodes is a set of players of the same group.

To complete the description of the normal-form of the network formation game, we have to specify the payoff functions. Therefore we consider the costs and benefits of the forming of links. Let $c_{i j}$ denote the costs to player $i$ of sponsoring a link to player $j$. We assume that these costs are increasing in the differences of the characteristics of the groups to which the two players belong. So, for two players $i \in N_{\ell}$ and $j \in N_{k}$, the cost of forming the link $(i, j)$ is given by

$$
c_{i j}=f(|l-k|),
$$

where $f(\cdot)$ is an increasing real-valued function in the length $|l-k|$ of the link. Observe that this modelling implies heterogeneity in costs due to fact that the costs of a link depend on its length. In fact, the costs are strictly increasing in the absolute value of the difference between the numbers of the (two) groups containing $i$ and $j$, i.e. links become more costly if the players are more different in their characteristics. We further assume that $f(0)>0$.

To obtain the benefits of the forming of links, it is useful to observe that any network induces an undirected graph on the set of players. Clearly, for each network $g \in \mathcal{G}$, the set $\bar{g}$ of undirected pairs of players defined by

$$
\bar{g}=\{\{i, j\} \subset N] \mid(i, j) \in g \text { or }(j, i) \in g\} .
$$




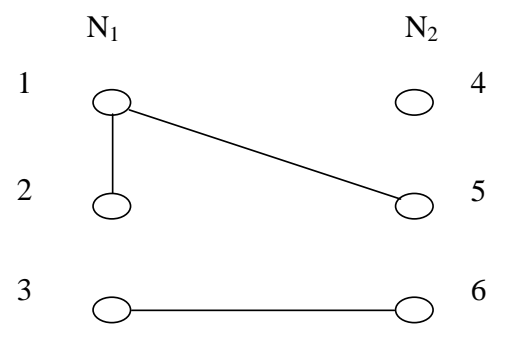

FiguRE $4 . \bar{g}=\{\{1,2\},\{1,5\},\{3,6\}\}$

gives an undirected graph on $N$ with an edge between any two players $i$ and $j$ if and only if the undirected pair $\{i, j\} \in \bar{g}$. For instance the network of Figure 3 gives the set of undirected pairs $\bar{g}=\{\{1,2\},\{1,5\},\{3,6\}\}$ and the resulting undirected graph is depicted in Figure 4.

Now, as usual in the network formation literature, player $i$ has access to the information of any player $j$ to which he is connected, including himself. In a network $g$, player $i$ is connected to player $j$ if there is at least one path between them, i.e. there exists a subset of players $j_{1}, \ldots, j_{r}$ with $i=j_{1}$ and $j=j_{r}$ such that $\left\{j_{k}, j_{k+1}\right\} \in \bar{g}$ for $k=1, \ldots, r-1$. It is assumed that player $i$ realizes a benefit equal to 1 from having access to the information from any player $j$ to which he is connected, including himself. Observe that the benefits are homogeneous and only depends on the number of players to which a player is connected. ${ }^{12}$

Now, the payoff of player $i$ in network $g$ is equal to the number of players to which $i$ is connected, including himself, minus the costs of the links sponsored by $i$. To state this formally, we introduce some notation. First, for $i \in N$, denote the set of players to whom player $i$ sponsors a link in network $g$ as

$$
N^{s}(i ; g)=\{j \in N \mid(i, j) \in g\} .
$$

Further, let $N_{k}^{s}(i ; g)=\left\{j \in N^{s}(i ; g) \mid j \in N_{k}\right\}$ be the set of players in group $k, k=1, \ldots, m$, to which player $i$ sponsors a link in network $g$. Next, for a network $g$, let $\widehat{g}$ be the collection of unordered pairs $\{i, j\}$ such that there exists a path between $i$ and $j$, thus

$$
\widehat{g}=\{\{i, j\} \subset N \mid \text { there is a path between } i \text { and } j \text { in } g\} .
$$

For example, in Figure 3 we have that $\widehat{g}=\{\{1,2\},\{1,5\},\{2,5\},\{3,6\}\}$. Likewise, let $N(i ; g)$ denote the set of players to which player $i$ is connected in $g$, including $i$ itself. So,

$$
N(i ; g)=\{j \in N \mid\{i, j\} \in \widehat{g}\} \cup\{i\} .
$$

\footnotetext{
${ }^{12}$ The normalization to one unit of benefit does not affect the results and simplifies the statements of our results.
} 
Now, for $i \in N_{\ell}$, the payoff function of player $i$ assigning the payoff to player $i$ in any network $g \in \mathcal{G}$ is given by

$$
\Pi_{i}(g)=|N(i ; g)|-\sum_{k=1}^{m}\left|N_{k}^{s}(i ; g)\right| f(|l-k|) .
$$

To avoid border problems, we assume that players lexicographically prefer sponsoring a link to not sponsoring a link. Thus if player $i$ receives the same payoff in $g \cup\{(i, j)\}$ as in $g$, then $i$ will strictly prefer $g \cup\{(i, j)\}$ above $g$. This completes the description of the stage game.

2.2. Nash networks. Given a network $g \in \mathcal{G}$, let $g_{-i}$ denote the network that remains when all of player $i$ 's links are removed, thus $g_{-i}=g \backslash g_{i}$. The network $g_{-i} \in \mathcal{G}$ can be seen as a strategy profile when $i$ chooses not to form a link with anyone. Now, a strategy $h_{i} \in \mathcal{G}_{i}$ is said to be a best reply of player $i$ to $g$ if for any $h_{i}^{\prime} \in \mathcal{G}_{i}$ holds that

$$
\Pi_{i}\left(h_{i} \cup g_{-i}\right)>\Pi_{i}\left(h_{i}^{\prime} \cup g_{-i}\right)
$$

or

$$
\Pi_{i}\left(h_{i} \cup g_{-i}\right)=\Pi_{i}\left(h_{i}^{\prime} \cup g_{-i}\right) \text { and }\left|h_{i}\right| \geq\left|h_{i}^{\prime}\right| .
$$

So, if $h_{i}$ is a best reply, then $h_{i}^{\prime}$ is also a best reply if both $\Pi_{i}\left(h_{i} \cup g_{-i}\right) \geq$ $\Pi_{i}\left(h_{i}^{\prime} \cup g_{-i}\right)$ and $\left|h_{i}\right| \geq\left|h_{i}^{\prime}\right|$ hold with equality. If there is at least one strict inequality, then $h_{i}^{\prime}$ is not a best reply. The set of all best replies of player $i$ to $g$ is denoted by $B_{i}(g)$ and a specific element of $B_{i}(g)$ will be denoted by $b_{i}(g)$. Observe that $B_{i}(g)=B_{i}\left(g_{-i}\right)$ for all $g$. Without any confusion we will use both notations.

Before we continue, we should emphasize some aspects of the best reply sets of the players. Consider network $g$ and let the network $g_{-i}$ consist of $k+1$ components $^{13}$, say $C_{0}, \ldots, C_{k}$. Without loss of generality we may assume that $i$ belongs to $C_{0}$. Let $b_{i}(g) \in B_{i}(g)$ be a best reply of player $i$ to $g_{-i}$. Then $b_{i}(g)$ does not contain a link $(i, j)$ to any player $j$ in his own component $C_{0}$, while there is at most one link in $b_{i}(g)$ to any other component. Further, if $i$ prefers to sponsor a link to some component $C_{h}$, $h=1, \ldots, k$, then he is indifferent between the players in that component that are closest to him and he will not sponsor a link to any other player of that component. Lastly, since the benefits of linking to some component are independent of the other links of the player, he will make his choice with respect to which of the players closest to him in this component irrespective of his links to other components. From this we obtain without further proof the following lemma ${ }^{14}$, which will appear useful later.

\footnotetext{
${ }^{13}$ See section 2.4 for the definition of a component.

${ }^{14}$ With slight abuse of usual notation, for $m$ non-empty sets $A_{h}, h=1, \ldots, m$, we define the product set as $\Pi_{h=1}^{m} A_{h}=\left\{\left\{a_{1}, \ldots, a_{m}\right\} \mid a_{h} \in A_{h}, h=1, \ldots, m\right\}$, i.e. the product set is the collection of all sets of $m$ elements containing precisely one element of each set $A_{h}$.
} 
Lemma 1. Let $g$ be a network in $\mathcal{G}$ and let $C_{1}, \ldots, C_{k}$ be the components of $g_{-i}$ not containing player $i$. When $i$ prefers to sponsor a link to $C_{h}$, let $D_{h}$ be the subset of players in $C_{h}$ that are closest to $i$; otherwise let $D_{h}=\emptyset$. Then $B_{i}(g)=\{\emptyset\}$ if $D_{h}=\emptyset$ for all $h$ and

$$
B_{i}(g)=\Pi_{\left\{h \mid D_{h} \neq \emptyset\right\}} D_{h} \text {, otherwise. }
$$

So, the lemma says that the best reply set contains $b_{i}=\emptyset \subset \mathcal{G}_{i}$ as its unique element when $i$ does not want to sponsor any link. Otherwise, a strategy $b_{i} \in \mathcal{G}_{i}$ is in the best reply set $B_{i}(g)$ if and only if $b_{i}$ contains precisely one link to a player in $D_{h}$ for any component $C_{h}$ with $D_{h} \neq \emptyset$. For example, let $g_{-i}$ consist of four components: $C_{0}, C_{1}, C_{2}$ and $C_{3}$. with $i \in C_{0}$. Further, suppose that $i$ prefers to sponsor a link to $C_{1}$ and $C_{2}$, but not to $C_{3}$. Let players $j, j^{\prime}, j^{\prime \prime}$ be the players in $C_{1}$ closest to $i$ and $k, k^{\prime}$ the players closest to $i \in C_{2}$. Then

$$
B_{i}(g)=\left\{b_{i} \in \mathcal{G}_{i} \mid b_{i}=\left\{\left(i, j^{*}\right),\left(i, k^{*}\right)\right\}, j^{*} \in\left\{j, j^{\prime}, j^{\prime \prime}\right\}, k^{*} \in\left\{k, k^{\prime}\right\}\right\} .
$$

Definition 1. A network $g$ is a Nash network if and only if $g_{i} \in B_{i}(g)$ for each $i$. A Nash network is a strict Nash network if $B_{i}(g)=\left\{g_{i}\right\}$ for all $i$, otherwise, a Nash network is said to be weak.

So, in a Nash network each player plays a best reply. In a strict Nash network $g$ it holds for any player $i$ that each other strategy $h_{i}$ gives a lower payoff to $i$ or it gives the same payoff but $h_{i}$ contains less links than $g_{i}$.

2.3. The Dynamics. To specify the dynamics of the model, we assume that time is discrete. Moreover, we assume that in every period each player plays the same strategy as he did the period before with probability $1-p$. However with positive probability $p<1$, a player receives the opportunity to update (revise) his strategy. The probability $p$ is assumed to be identical for each player and the probability that player $i \in N$ is allowed to update his strategy does not depend on the fact whether or not player $j \in N \backslash\{i\}$ is allowed to update. This means that in each period, the set of players that is allowed to update, can be any one subset of the population. If a player updates, he will act according to a pre-specified behavioral rule. We assume that players are myopically rational, that is, whenever players update their strategy they play a best reply (because they are rational) to the current network (because they are myopic). Thus a player does not take into account that also other players will update their strategies. If a player has several best replies, then he chooses one according to a random device that assigns positive probability to each best reply. The resulting dynamics is the socalled best reply dynamics. Because $p$ is smaller than 1 , not every player receives the chance to update every turn. This feature of the model is called inertia. Lastly, we do not allow for any perturbations in the dynamics: no player ever plays a strategy that does not follow from the behavioral rule. Summarizing, we use an unperturbed best reply dynamics with inertia. 
Formally, the dynamics induces a Markov process on the state space $\mathcal{G}$ of all networks. More precisely, for $g \in \mathcal{G}$, let $\phi_{g}^{i}$ be a probability distribution on $\mathcal{G}_{i}$ assigning positive probability to $h_{i} \in \mathcal{G}_{i}$ if and only if $h_{i} \in B_{i}(g)$. So the behavioral rule of every player is given by these probability distributions in the set $G$ of all networks. Now, starting from some initial network $g^{0}$ at time $t=0$, let the Markov process be given by the sequence of random variables $X_{t}, t \geq 1$, on the state space $\mathcal{G}$. Then the time-independent transition matrix of transition probabilities $P_{g h}$ that at time $t+1$ the process moves to state $h \in \mathcal{G}$ when it was in state $g \in \mathcal{G}$ at time $t$ is given by

$$
P_{g h}=\operatorname{Prob}\left\{X_{t+1}=h \mid X_{t}=g\right\}=\times_{i \in N} P_{g_{i}, h_{i}}^{i},
$$

where $P_{g_{i}, h_{i}}^{i}$ is the probability that player $i$ updates to strategy $h_{i}$ when he played $g_{i}$, i.e.

$$
P_{g_{i}, h_{i}}^{i}=\left\{\begin{array}{l}
p \times \phi_{g}^{i}\left(h_{i}\right) \text { when } h_{i} \in B_{i}(g) \text { and } h_{i} \neq g_{i}, \\
1-p+p \times \phi_{g}^{i}\left(h_{i}\right) \text { when } h_{i} \in B_{i}(g) \text { and } h_{i}=g_{i}, \\
1-p \text { when } h_{i}=g_{i} \text { and } g_{i} \notin B_{i}(g) \\
0, \text { otherwise. }
\end{array}\right.
$$

Starting from some initial state $g^{0}$ at time $t=0$, the Markov process generates a sequence of networks. If at time $t$ the network is $g^{t}$, then the set of networks that are possible at time $t+1$ is given by

$$
\mathcal{G}^{t+1}=\left\{g \in \mathcal{G} \mid g_{i} \in B_{i}\left(g^{t}\right) \cup g_{i}^{t}, \text { for all } i \in N\right\} .
$$

It is well-known that the process converges to some recurrent class of states. To characterize the networks which may appear in a recurrent class of states, we will use the concept of minimal curb set, to be introduced in the next section.

2.4. Additional notation and terminology. In this subsection we introduce some more notation and terminology that we will need in the sequel of this paper. First, we state the well-known concepts of components, minimality, connectedness, cycles and empty networks. Second, we introduce some specific notions that will used in the next sections, such as internal links and external links. Finally, we define some sets of players in relation to a link that a player sponsors.

A component of a network $g$ is a maximally connected subset of players, i.e. the subset is connected and becomes unconnected when adding any other player to the subset. Let $C(g)=\left\{C_{1}, \ldots, C_{\gamma(g)}\right\}$ be the set of components of a network $g$, where $\gamma(g)$ is the number of components in network $g$. Observe that every player belongs to exactly one component, so that the components form a partition of the set $N$ of players. We denote the number of components that have at least one member within a certain group, say group $\ell$, by $\gamma_{\ell}(g)$. Formally:

$$
\gamma_{\ell}(g)=\left|\left\{C_{k} \in C(g) \mid N_{\ell} \cap C_{k} \neq \emptyset\right\}\right| .
$$


For example, in Figure 3 there are 3 components: $\{1,2,5\},\{3,6\}$ and $\{4\}$, while $\gamma_{1}(g)=2$ and $\gamma_{2}(g)=3$. In the network $g$ of Figure 8 there is only one component and thus $\gamma_{\ell}(g)=1$ for any group $\ell$.

A network $g$ is said to be minimal if and only if $\gamma(g)<\gamma(g \backslash\{(i, j)\})$ for all $(i, j) \in g$. Thus a network is minimal if removing any link would break the connection between some pair of connected players. Clearly, a network is minimal if and only if there is a unique path between every pair of connected players (there are no 'cycles').

We say that a network $g$ is connected if every player is connected to every other player. Thus a connected network consists of only one component. If a connected network is minimal then we call it minimal connected. It follows that any link in a minimal connected network is critical in the way that the network breaks up into two components when deleting any link. A cycle in a network $g$ is a path of $k \geq 2$ different unordered pairs $\left\{i_{1}, i_{2}\right\}, \ldots,\left\{i_{k}, i_{k+1}\right\}$ in the induced undirected network (graph) $\bar{g}$ such that all players on the path are different, except that $i_{1}=i_{k+1}$. Thus in a network $g$ on the players set $N=\{1,2,3,4,5\}$, both the set of links $\{(1,2),(2,3),(1,3)\}$ and $\{(4,5),(5,4)\}$ constitute a cycle. Notice that a network contains a cycle if and only if it is not minimal. A network without links is called an empty network. By definition such a network is minimal and consists of $n$ components.

Next we introduce some concepts with respect to the groups in the model. A link $(i, j)$ is called internal if $i$ and $j$ belong to the same group. Otherwise we say that the link $(i, j)$ is external. We say that $i, j \in N_{\ell}$ are internally connected in $g$ if there exists a path in $g$ between $i$ and $j$ that only contains internal links. We say that $i, j \in N_{\ell}$ are externally connected if they are connected but not internally connected. Group $\ell$ is called entirely internally connected in network $g$ if all pairs $i, j \in N_{\ell}$ are internally connected, group $\ell$ is called entirely externally connected if all pairs $i, j \in N_{\ell}$ are externally connected. We denote the set of entirely externally connected groups in $g$ by $\mathcal{N}^{E}(g)$, and the set of entirely internally connected groups by $\mathcal{N}^{I}(g)$. Notice that $\mathcal{N}^{I}(g) \cup \mathcal{N}^{E}(g) \subseteq \mathcal{N}=\left\{N_{1}, \ldots, N_{m}\right\}$, for all $g \in \mathcal{G}$. We say that the set of players $N_{\ell} \in \mathcal{N}$ in group $\ell$ is a center-sponsored star if and only if there is one player in $N_{\ell}$ that sponsors a link to each other player of $N_{\ell}$, and no other player in $N_{\ell}$ sponsors any internal link. An example of a center-sponsored star is group 3 in the network of Figure 7. Finally, for $g \in \mathcal{G}$, we denote the number of internal links within group $\ell$ by $\sigma_{\ell}(g)$ and the total number of internal links within the network $g$ by $\sigma(g)$, thus $\sigma(g)=\sum_{\ell=1}^{m} \sigma_{\ell}(g)$. For example in Figure $3 \sigma_{1}(g)=2, \sigma_{2}(g)=0$, and $\sigma(g)=2$.

Next, we introduce some definitions of sets of players that a player observes via a link that he sponsors. At the end we give an example to clarify these definitions. First, for a network $g \in \mathcal{G}$ and $i \in N$, we denote $A_{i i^{\prime}}(g)$ by the set of players that $i$ observes exclusively via his link to some player 
$i^{\prime} \in N \backslash\{i\}$ by

$$
A_{i i^{\prime}}(g)=\left\{j \in N \mid j \in N(i ; g) \text { and } j \notin N\left(i ; g \backslash\left\{\left(i, i^{\prime}\right)\right\}\right)\right\} .
$$

Thus $A_{i i^{\prime}}(g)$ is the set of players that $i$ observes in $g$ and that $i$ would not observe if $i$ deletes his link to $i^{\prime}$. Observe that $A_{i i^{\prime}}(g)=\emptyset$ if $\left(i, i^{\prime}\right) \notin g$. We proceed by defining $A_{i i^{\prime}}^{E}(g)$ as the set of players in $A_{i i^{\prime}}(g)$ that are members of entirely externally connected groups that are not fully observed by $i$ via $\left(i, i^{\prime}\right)$, i.e.

$$
A_{i i^{\prime}}^{E}(g)=\left\{j \in A_{i i^{\prime}}(g) \mid j \in N_{\ell}, N_{\ell} \in \mathcal{N}^{E}(g) \text { and } A_{i i^{\prime}}(g) \cap N_{\ell} \neq N_{\ell}\right\} .
$$

Thus $A_{i i^{\prime}}^{E}(g)$ consists of all players that satisfy three conditions: first, each player must also be part of $A_{i i^{\prime}}(g)$. Second, each player must be part of an entirely externally connected group. Third, there must be another player in the same group that is not part of $A_{i i^{\prime}}(g)$. Conversely, let $D_{i i^{\prime}}^{E}(g)$ denote the set of players in entirely externally connected groups such that other players of that group are observed by $i$ via $\left(i, i^{\prime}\right)$, but they themselves are not. Thus:

$$
D_{i i^{\prime}}^{E}(g)=\left\{j \in N \backslash A_{i i^{\prime}}(g) \mid j \in N_{\ell}, N_{\ell} \in \mathcal{N}^{E}(g) \text { and } A_{i i^{\prime}}(g) \cap N_{\ell} \neq \emptyset\right\} .
$$

Observe that if there exists a player $j \in A_{i i^{\prime}}^{E}(g) \cap N_{\ell}$, then each player in $N_{\ell}$ is either part of $A_{i i^{\prime}}^{E}(g)$ or of $D_{i i^{\prime}}^{E}(g)$. Finally, let $N^{r}(i ; g)$ denote the set of players that sponsor a link to player $i$ in network $g$, i.e.

$$
N^{r}(i ; g)=\{j \in N \mid(j, i) \in g\}
$$

and for $i \in N_{\ell}$, let $N^{R}(i ; g)$ be the set of players that sponsor a link to $i$, such that any $i^{\prime} \in N_{\ell}$ observed by $i$ will also be observed by $i$ when any $j \in N^{R}(i ; g)$ deletes his link $(j, i)$, i.e.

$$
N^{R}(i ; g)=\left\{j \in N^{r}(i ; g) \mid \gamma_{\ell}(g)=\gamma_{\ell}(g \backslash\{(j, i)\})\right\} .
$$

As an example, consider Figure 8, but with links $(2,1)$ and $(2,3)$ replaced by $(5,2)$ and $(6,3)$. Hence $N_{1} \subseteq \mathcal{N}^{E}(g)$, i.e. group 1 is entirely externally connected. Player 12 observes players 1 to 6 and player 9 via the link $(12,9)$. Moreover if we would remove $(12,9)$ from this network, then player 12 observes none of these players. Therefore $A_{12,9}(g)=\{1,2,3,4,5,6,9\}$. Similarly $A_{5,4}(g)=\{4\}$. If we would add the link $(4,5)$ to the network, then $A_{5,4}(g \cup\{(4,5)\})=\emptyset$, since player 5 would also observe player 4 via $(4,5)$. Let us now find $A_{12,9}^{E}(g)$. In $A_{12,9}(g)$, the players $1,2,3$ and 9 belong to entirely externally connected groups. However, all members of group 1 belong to $A_{12,9}(g)$, thus $A_{12,9}(g) \cap N_{1}=N_{1}$. On the other hand, the intersection of $A_{12,9}(g)$ with group 3 is a strict subset of group 3 . Therefore players in $A_{12,9}(g)$ that are member of group 3 are part of $A_{12,9}^{E}(g)$, while members of groups 1 and 2 are not: $A_{12,9}^{E}(g)=\{9\} . D_{12,9}^{E}$ are all players that are not part of $A_{12,9}^{E}(g)$, but that belong to a group of which some players are part of $A_{12,9}^{E}(g)$. In this network $D_{12,9}^{E}(g)=\{7,8,10\}$. Finally, 
note that $N^{r}(9 ; g)=\{5,12\}$ and that $\gamma_{3}(g)=\gamma_{3}(g \backslash\{(5,9)\})=1$, while $\gamma_{3}(g \backslash\{(12,9)\})=2$. Therefore $N^{R}(9, g)=\{5\}$.

\section{Super Tight Curb Sets}

Standard theory shows that strict Nash equilibria are stable in a best reply dynamic process. A strict Nash equilibrium is characterized by a strategy profile in which each agent is playing his unique best reply. So, if the dynamics at some time $t$ reaches such a state and the dynamics is unperturbed, then it will stay in such a state forever. In other words, a strict Nash equilibrium is an absorbing state (a state that is not 'left' once it is reached) of an unperturbed best reply dynamics.

In general, the unperturbed best reply dynamics converges to a recurrent class of states. A class of states is recurrent when (i) the dynamics stays within this class forever as soon as it has reached one of the states of the class, and (ii) from each state in the class there is a positive probability to reach any other state in the class within a finite number of steps. For example consider the bimatrix game of Figure 5. It represents a simultaneous move game of two players with strategy space $\mathcal{S}=\mathcal{S}_{1} \times \mathcal{S}_{2}$, where $\mathcal{S}_{1}=\{a, b, c, d, e\}$ is the strategy space of player 1 and $\mathcal{S}_{2}=\{A, B, C, D, E\}$ the strategy space of player 2 . We now suppose that the game is repeated infinitely and that the players revise their strategies conform the unperturbed best reply dynamics that we defined in the previous section. The state space of the dynamics is the strategy space $\mathcal{S}=\mathcal{S}_{1} \times \mathcal{S}_{2}$. We denote a state, or strategy profile, by $s=\left(s_{1}, s_{2}\right) \in \mathcal{S}$. There are three recurrent classes of states, namely $X=\{(a, A),(a, B),(b, A),(b, B)\}, Y=\{(c, C),(c, D),(d, C),(d, D)\}$ and $Z=\{(e, E)\}$.

Clearly, the strategy profile $s=(e, E)$ in the singleton recurrent class $Z$ is a strict Nash equilibrium. Hence when the dynamics reaches this state, then it will stay there forever. Any state in the class $X$ in a weak Nash equilibrium. In this class the dynamics can go from one state to any other state within one step. For instance, consider $s=(a, A) \in X$. In this state, player 1 is indifferent between playing $a$ and playing $b$ and player 2 is indifferent between $A$ and $B$. Therefore, when the dynamics is in state $(a, A)$, it goes to $(a, B)$ or $(b, A)$ when one of the players updates, or to $(b, B)$ when both players revise their strategies simultaneously.

Within the recurrent class $Y$ the dynamics can go from one state to precisely one other state. For instance, when the dynamics is in $(c, C)$, it goes to $(c, D)$ when player 2 updates (and stays otherwise in $(c, C)$ ), from $(c, D)$ to $(d, D)$ when player 1 updates, from $(d, D)$ to $(d, C)$ when player 2 updates and then back to $(c, C)$ when player 1 updates. So, in this case there is a unique cycle containing all the states of $Y$ along which the dynamics moves. Observe, that none of these states is a Nash equilibrium. Nevertheless, this recurrent class will be reached when player 2 updates and player 1 plays $c$ of $d$, or when player 1 updates and player 2 plays $C$ or $D$. Apparently, it may happen quite well that the dynamics will reach this recurrent class of 


\begin{tabular}{|c|c|c|c|c|c|c|}
\hline players & \multicolumn{6}{|c|}{ Player 2} \\
\hline \multirow{6}{*}{$\begin{array}{l}\overrightarrow{\bar{d}} \\
\overrightarrow{\vec{a}}\end{array}$} & strategies & $\mathrm{A}$ & B & $\mathrm{C}$ & $\mathrm{D}$ & $E$ \\
\hline & $\mathrm{a}$ & 3,5 & 2,5 & 2,4 & 2,4 & 0,0 \\
\hline & $\mathrm{b}$ & 3,4 & 2,4 & 2,3 & 2,3 & 0,0 \\
\hline & $\mathrm{c}$ & 2,1 & 1,1 & 4,2 & 3,5 & 0,0 \\
\hline & $\mathrm{d}$ & 2,1 & 1,1 & 3,5 & 4,2 & 0,0 \\
\hline & $\mathrm{e}$ & 0,0 & 0,0 & 0,0 & 0,0 & 2,2 \\
\hline
\end{tabular}

FiguRe 5. 2-player 5x5 game

states. This makes that each of the three recurrent classes is a candidate solution for the best reply dynamics.

To be able to characterize the states which may occur in a recurrent class of the unperturbed best reply dynamics of the network formation game, we will consider the static solution of the so-called curb sets, introduced by Basu and Weibull (1991). A set of strategy profiles is said to be closed under rational behavior (curb) if it contains all its best replies. Formally, let be given a game with players set $N=\{1, \ldots, n\}$ and strategy space $\mathcal{S}=\times_{i \in N}$ $\mathcal{S}_{i}$, where $\mathcal{S}_{i}$ is the strategy set of player $i$. Then a subset $S \subseteq \mathcal{S}$ is a curb set if

$$
B(S)=\times_{i \in N} \cup_{s \in S} \quad B_{i}(s) \subseteq S,
$$

where $B_{i}(s)$ is the $i$-th player's best reply set to strategy profile $s$. Clearly, any strict Nash equilibrium is a (singleton) curb set. So, the recurrent class $Z$ in the example of Figure 5 is a curb set. It is easily seen that also the recurrent classes $X$ and $Y$ are curb sets. However, by definition, for a game with bounded strategy spaces also the whole strategy space of the game is a curb set, so that the concept of curb set is not very restrictive. Therefore, often additional properties are imposed to obtain an appropriate solution concept.

A curb set $S \subseteq \mathcal{S}$ is said to be tight if $B(S)=S$ and $S \subseteq \mathcal{S}$ is said to be a minimal curb set (MCS) if it does not contain a strict subset $S^{\prime} \subset S$, such that $B\left(S^{\prime}\right) \subseteq S^{\prime}$. Notice that if $S$ is an MCS, then $B(S)=S$, i.e. all minimal curb sets are tight. Clearly, each strict Nash equilibrium constitutes a singleton MCS. In the game of Figure 5, all three recurrent classes $X, Y$ and $Z$ are minimal curb sets. It follows straightforwardly that a recurrent class is always contained in a minimal curb set. However, in general the reverse does not hold. For instance, consider the bimatrix game given in Figure 6.

In this game, there is only one minimal curb set, namely the whole strategy space $\mathcal{S}=\{a, b, c\} \times\{A, B, C\}$ itself. So, the unique minimal curb set contains all possible nine strategy profiles. However, the unique recurrent class of the unperturbed best reply dynamics contains only six states and 


\begin{tabular}{|l|c|c|c|c|}
\hline players & \multicolumn{3}{|c|}{ Player 2 } \\
\hline \multirow{3}{*}{$\overrightarrow{\bar{D}}$} & strategies & A & B & C \\
\cline { 2 - 5 } & $\mathrm{a}$ & 2,4 & 1,1 & 4,2 \\
\cline { 2 - 5 } & $\mathrm{b}$ & 4,2 & 2,4 & 1,1 \\
\cline { 2 - 5 } & $\mathrm{c}$ & 1,1 & 4,2 & 2,4 \\
\hline
\end{tabular}

FiguRE 6. 2-player 3x3 game

is given by the set $\{(a, A),(b, A),(b, B),(c, B),(c, C),(a, C)\}$. Once the dynamics reaches one of these states, it will cycle along these states forever. So, the unique recurrent class is a real subset of the unique minimal curb set, but is not equal to this MCS. The following theorem states that every minimal curb set contains at least one recurrent class of the best reply dynamic defined in Subsection 2.3.

Theorem 1. Consider a game with population $N=\{1, \ldots, n\}$, with strategy space: $\mathcal{S}=\times_{i \in N} \mathcal{S}_{i}$. Then each minimal curb set contains at least one recurrent class of the dynamic defined by Equation 2.1, with $\mathcal{G}=\mathcal{S}$.

Proof Note first that if $s^{t}$ is the state at time $t$, then the set of possible states in time $t+1$ is given by $\mathcal{S}^{t+1}=\mathcal{G}^{t+1}$ as given by Equation 2.2, i.e.

$$
\mathcal{S}^{t+1}=\left\{s \in \mathcal{S} \mid s_{i} \in B_{i}\left(s^{t}\right) \times s_{i}^{t}, \text { for all } i \in N\right\} .
$$

Second, observe that if $X$ is some minimal curb set, then $B(X) \times X \subseteq X$. Therefore, if $s^{t} \in X$, then $s^{T} \in X$ for all $T \geq t$. This implies that $X$ contains at least one recurrent class of the dynamics.

Notice that we do not claim that any recurrent class is contained by some minimal curb set.

To obtain a further refinement of the concept of curb set, observe that the curb set $X$ in the example of Figure 5 has the special property that the best reply of player $i, i=1,2$ to a strategy profile $s \in X$ is independent of $s$, i.e. for any $s \in X$ we have that $B_{1}(s)=\{a, b\}$ and $B_{2}(s)=\{A, B\}$. This property trivially also holds for $Z$, but it does not hold for $Y$. For the latter set we have that $B_{1}(c, C)=B_{1}(d, C)=\{c\}$ and $B_{1}(c, D)=B_{1}(d, D)=\{d\}$. According to GGK, an MCS $S$ satisfying the property that for any player $i$ the best reply set is the same for any strategy profile $s \in S$ is called a Super Tight Curb Set (STCS). Formally, for $S \subseteq \mathcal{S}$ and $i \in N$, let $S_{i}$ be the restriction of $S$ to $\mathcal{S}_{i}$, i.e.

$$
S_{i}=\left\{s_{i} \in \mathcal{S}_{i} \mid \exists \widehat{s} \in S \text { with } \widehat{s}_{i}=s_{i}\right\} .
$$

Then a curb set $S$ is STCS when $S$ is a minimal curb set and it holds that $B_{i}(s)=S_{i}$ for any $s \in S$. So, within a STCS the best reply set of any player is independent of the strategy chosen by any other player. The STCS property implies two things. First, if $S$ is STCS, then any strategy profile $s \in S$ is a Nash equilibrium. Clearly, since $B_{i}(s)=S_{i}$ for all $s \in S$ it holds 
that $s \in S=B(s)$. Second, when $S$ is STCS, then the best reply dynamics can go from any state in $S$ to any other state in $S$ within one step, since all players can update simultaneously. So, when the dynamics is in some state $s \in S$ at time $t$, then with positive probability the dynamics can be in any state $\widehat{s} \in S$ at time $t+1$. Consequently, when $S$ is a STCS, then $S$ is a recurrent class of states. Concluding we have that any recurrent class is contained in an MCS, while on the other hand an MCS is a recurrent class if it is STCS.

Theorem 2. Consider a game with population $N=\{1, \ldots, n\}$, with strategy space: $\mathcal{S}=\times_{i \in N} \mathcal{S}_{i}$. Let the dynamics be defined by Equation 2.1, with $\mathcal{G}=\mathcal{S}$. Then a minimal curb set that is super tight is identical to the unique recurrent class of the dynamic it contains.

In the examples given above we have that the recurrent classes $X$ and $Z$ of Figure 5 are STCS, whereas the recurrent class $Y$ is an MCS, but not STCS. The unique recurrent class of Figure 6 is a real subset of the unique MCS and therefore it is not an STCS.

We now return to our Multiple Group network formation game. Notice that Theorems 1 and 2 apply to this model. For a subset of strategy profiles $X$ of the space $\mathcal{G}$ of all networks, we have that

$$
\begin{gathered}
X_{i}=\left\{g_{i} \mid g \in X\right\}, \\
B_{i}(X)=\cup_{g \in X} B_{i}(g),
\end{gathered}
$$

and

$$
B(X) \equiv\left\{g \in \mathcal{G} \mid g=\cup_{i \in N} g_{i}, g_{i} \in B_{i}(X) \text {, for all } i\right\} .
$$

A curb set $X$ is STCS if $X$ is minimal and $B_{i}(g)=X_{i}$ for all $g \in X$ and all $i \in N$. Two examples of minimal curb sets for the network formation game with four groups are given in the Figures 7 and 8 . The network of Figure 7 is a strict Nash equilibrium. The network of Figure 8 however is element of a minimal curb set that contains 12 networks (states). Observe that in this figure any player, except player 5 , has a unique best reply and that this best reply is identical to their current strategy. Player 5 has 12 best replies (3 alternatives to group 1 times 4 alternatives to group 3), including his current strategy, playing link $(5,9)$, itself. For example, whether 5 links to $7,8,9,10,11,12$, or 13 , he will observe the same set of players. However the links to the players in group 4 are more expensive than the links to group 3 . Therefore there are 3 alternatives for the link $(5,9)$. So, since any player plays a best reply, the network is a Nash network.

Further observe that the sets of best replies of the other players are unaffected by the particular best reply chosen by 5 . For instance, although the value of the link $(12,9)$ decreases if 5 switches from 9 to 8 , the value is still higher than the cost of the link, since $f(1) \leq 1$ and therefore it is still optimal for player 12 to play this link. Since the best reply set of any 


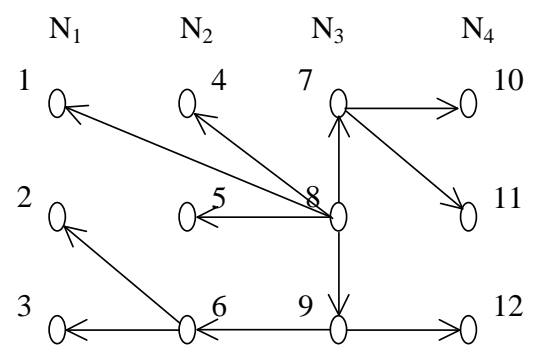

Figure 7. Strict Nash equilibrium for $f(2) \leq 1$.

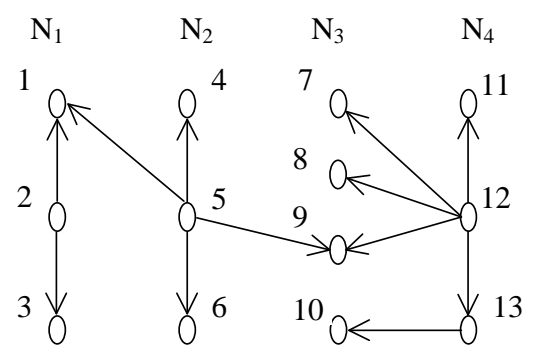

FiguRE 8. Network in a non-singleton MCS for $f(1) \leq 1$.

player other than 5 is singleton, the best reply set of each player $i \in N$ is not affected by the particular best reply played by $j \in N \backslash\{i\}$. Therefore the minimal curb set containing the network of 8 is STCS and thus a recurrent class of states. Hence, any network in this curb set is a Nash network and the best reply dynamics may go from any network in the set to any other network in the set within one step. In particular in should be noticed that in an STCS of networks, the set of best replies is unaffected by any sequence and any timing in which any set of players choose to select any of their alternative best replies. Also notice that, as proved by Bala and Goyal (2000), all Nash networks are minimal.

\section{Super Tight Curb Sets in the MG model}

In the next two sections we want to characterize the networks that are likely to arise in the MG model. We focus to the case where $f(0) \leq 1$ and $f(1) \leq \max _{\ell}\left|N_{\ell}\right|$. The analysis can be extended to all cost functions, and theorem 5 will continue to hold. The proof for this is not included in the paper, because it is cumbersome and large, while it adds little additional insight.

GGK found that the strict Nash equilibrium does not exist for all parameters, and that it is not the only type of network that survives the best reply 
dynamics. Hence the focus on strict Nash equilibria appears overly restrictive. However, as in most network formation models, the concept of weak Nash equilibrium has little bite: we cannot say much about such networks. Moreover, most Nash equilibria do not survive the best reply dynamics. Hence we use an alternative solution concept: the minimal curb set. As we show in this section, the minimal curb sets of this model contain only Nash equilibria, namely those that belong to a recurrent class. Furthermore, all networks in a recurrent class belong to a minimal curb set. Therefore the minimal curb set is, in this model, a good refinement of the set of Nash equilibria. In Section 5 we give necessary and sufficient conditions for networks to belong to a minimal curb set.

We prove these results in Theorem 5 . There we show that every recurrent class contains a subset of networks that is, by itself, a super tight curb set. Together with Theorems 1 and 2 it follows that every minimal curb set is super tight. This enables us to characterize the minimal curb sets, which we do in Section 5. Moreover, since the dynamics have at least one recurrent class, this also proves the existence of a Nash network in the network formation game.

To obtain the main results of this section that any MCS is STCS, we first prove two other theorems. These theorems state that for certain types of networks at most one cycle can arise if two players update simultaneously. Recall that for a network $g \in \mathcal{G}$ of directed pairs of players, $\bar{g}$ is the corresponding undirected graph on $N$ and $\widehat{g}$ the collection of all pairs connected to each other in $g$ through a path.

Theorem 3. Let $g \in \mathcal{G}$ be connected and minimal. If exactly two agents update simultaneously, then there is at most one cycle in the resulting network $g^{\prime}$.

Proof Let the two updating players be $i$ and $j$. Because the network is minimal and connected, neither player will sponsor more links in the network $g^{\prime}$ after updating than in the network $g$ before updating. Otherwise, they would expect to create a cycle, which contradicts that the players play a best reply to $g$ when updating. Thus the number of links does not increase. Suppose that $g^{\prime}$ contains a cycle. Since the number of links did not increase, this implies that $g^{\prime}$ is not connected. Further, since only $i$ and $j$ have been updating, the cycle contains at least one of them. W.l.o.g. assume that $i$ is in the cycle and let $N_{\text {con }}^{i} \subseteq N$ be the set of players connected to $i$ in $g^{\prime}$, including $i$ itself. We first show that $j \in N_{\text {con }}^{i}$. Suppose not. Clearly, the cycle can only contain players in $N_{c o n}^{i}$. However, if $j \notin N_{c o n}^{i}$ then all players in $N_{\text {con }}^{i}$ except $i$ itself play in $g^{\prime}$ the same strategy as in $g$. Since $g$ did not contain a cycle, this means that $i$ sponsors at least one link of the cycle, which contradicts that in $g^{\prime} i$ plays a best reply to $g$. Hence $j \in N_{c o n}^{i}$.

Suppose player $i$ and $j$ deleted together $x$ links. Then if no cycles would have been created there would have been $x+1$ components, because the total number of links in the network is now $|N|-x-1$. In each cycle, any 
one link could be deleted without removing the connection (path) between any two players. Therefore, such a link does not connect any two, otherwise disconnected players. Therefore, per cycle in $g^{\prime}$ there is one more component in $g^{\prime}$, which does not contain $i$ and $j$. Let us denote any one such component by $N_{i s o l}^{\prime} \subset N$. Since $g$ was connected, either $i$ or $j$ or both sponsored a link to a player of $N_{i s o l}^{\prime}$ in $g$. Without loss of generality, suppose that $i$ sponsored a link in $g$ to some player $i^{\prime} \in N_{i s o l}^{\prime}$. Since the players in $N_{i s o l}^{\prime}$ did not update and $N_{i s o l}^{\prime}$ is connected, this implies player $i$ observed all players in $N_{i s o l}^{\prime}$ through his link to $i^{\prime}$, i.e. $N_{i s o l}^{\prime} \subseteq A_{i i^{\prime}}(g)$. Since $i$ played a best reply to $g$, player $i$ thought that he would still observe the players $N_{i s o l}^{\prime}$ in $g^{\prime}$. So, when updating player $i$ replaced his link $\left(i, i^{\prime}\right)$ by a link $\left(i, i^{\prime \prime}\right)$ such that $N_{i s o l}^{\prime} \subseteq A_{i i^{\prime \prime}}\left(g_{-i} \cup g_{i}^{\prime}\right)$. However, it appears that the players in $N_{i s o l}^{\prime}$ are not observed anymore, i.e. $N_{i s o l}^{\prime} \subseteq N \backslash A_{i i^{\prime \prime}}\left(g^{\prime}\right)$. Hence it follows that player $j$ has a link via which $i$ expected to access $N_{i s o l}^{\prime}$, i.e. there is a player $j^{\prime}$ such that $N_{i s o l}^{\prime} \subseteq A_{j j^{\prime}}(g)$. This implies that $i \in A_{j j^{\prime}}(g)$ and $j \in A_{i i^{\prime}}(g)$ and that both sets contain $N_{i s o l}^{\prime}$, i.e. in $g$ there is a path from $i$ to $j$ containing players of $N_{i s o l}^{\prime}$. Now, suppose there is a second component $N_{i s o l}^{\prime \prime} \neq N_{i s o l}^{\prime}$ of connected players in $g^{\prime}$ not containing $i$. Then $g$ also contains a path from $i$ to $j$ containing players of $N_{i s o l}^{\prime \prime}$. However, this contradicts that $g$ is minimal. So there are at most two components in $g^{\prime}$, one containing player $i$ and one not. Therefore $g^{\prime}$ can contain at most one cycle.

Theorem 4. Let $g \in \mathcal{G}$ be minimal and let $j \in N$ be such that $g_{j} \in B_{j}(g)$. Then if $i$ and $j$ simultaneously update, the resulting network $g^{\prime}$ will contain at most one cycle.

Proof Let $C$ be the component of the network $g$ that contains $j$. Because $j$ plays a best reply in $g$, apparently he strictly prefers not to sponsor a link to any player $h \notin C_{k}$. Thus if $j$ and a player $i \neq j$ simultaneously update, then any cycle involving any player $h \notin C$ is created by the best reply of $i$. However, this contradicts that $i$ plays a best reply to $g$. So, any cycle is created by $j$ and $i$ together and therefore can only include players of $C$. Therefore, for the purpose of determining the number of cycles that $g^{\prime}$ can contain, we can treat $C$ as a minimal connected network by itself and apply theorem 3. This concludes the proof.

\section{Example 1.}

Consider the network $g$ of Figure 9 with two groups with player $1 \in N_{1}$ and players $2,3,4,5 \in N_{2}$ and let the cost of any link be smaller than 1 . Then player 1 is indifferent between any link to one of the players 3,4 and 5 , while player 4 is indifferent between sponsoring a link to either player 2 or player 3. So, if both players update simultaneously then it may happen that player 4 replaces his link to 3 by a link to 2 and player 1 replaces his link to 3 by a link to 5 . So, in the resulting network $g^{\prime}$ the players 1 and 4 miscoordinate and neither player sponsors a link to 3 . As a result, in $g^{\prime}$ player 3 is isolated 


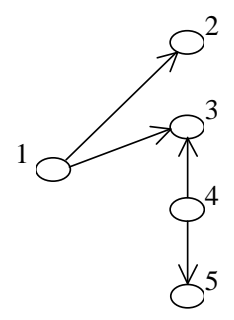

Figure 9. Example of miscoordination

and a cycle between 1, 2, 4 and 5 occurs. Observe that in $g^{\prime}$ the number of links is not larger than the number of links in $g$.

We are now ready to state the first of the two main theorems of this paper, saying that under certain conditions any recurrent class $(\mathrm{RC})$ of the network formation is also a super tight curb set.

Theorem 5. Let be given a network formation game with $m$ groups of players $N_{1}, \ldots, N_{m}, m \geq 2$ and $\left|N_{\ell}\right| \geq 2$ for all $\ell$, and with a strictly increasing cost function $f(\cdot)$ satisfying $f(0) \leq 1$ and $f(1) \leq \max _{\ell}\left|N_{\ell}\right|$. If $X \subseteq \mathcal{G}$ is a recurrent class from the dynamics specified in Equation 2.1, then $X$ is also an STCS.

The proof of this theorem follows from a number of lemmas stated below. The lemmas show that any network in some subset of an RC satisfies certain properties. At the end it follows that any network of an RC satisfies these properties, which implies that the RC contains an STCS. It then directly follows that the MCS is super tight and that each recurrent class is identical to some MCS. In the reasoning underlying the lemmas, it should be kept in mind that, first, if a network is in some $\mathrm{RC} X$, any sequence of updates generates a sequence of networks in $X$; second, if a network is minimal and only one player updates, then the resulting network is also minimal; and third, if a network is minimal and any number of players update simultaneously, then the resulting network contains at least the same number of internal links. As far as needed we refer to Appendix A for the proofs of the lemmas.

As we have seen any network in a super tight curb set is a Nash network and thus is a minimal network. We therefore first adapt the result from Bala and Goyal (2000), saying that any RC contains at least one minimal network. To see this, let $X$ be an RC, $g^{0}$ a network in $X$, and suppose that all players update subsequently, i.e. each player $i$ plays subsequently a best reply to network $g^{i-1}$, where $g^{i}$ is the network that occurs after the update of player $i, i=1, \ldots, n$. Clearly, the resulting network $g^{n}$ has to be minimal. If not, then there must be a cycle in the network. But then the last player $i$ contained in this cycle that sponsors a link in the cycle, did not play a best reply to $g^{i-1}$ : a contradiction. Since by definition each network 
$g^{i}$ is in $X$, we obtain that $X$ must contain a minimal network. So, without further proof we can state the following lemma.

Lemma 2. Let $X$ be an $R C$. Then there exists a network $g \in X$ that is minimal.

Next we define for an MCS $X$ the non-empty subset of minimal networks obtained by maximizing lexicographically the number of links from length zero to length $m-1$. More precisely, for $X \subseteq \mathcal{G}$ a recurrent class, define

$$
X^{\min }=\{g \in X \mid g \text { is minimal }\} .
$$

From Lemma 2 we know that $X^{\text {min }}$ is not empty. Further, let $\delta_{k}(g)$ be the number of links of length $k$ in network $g$. Then we define successively for $k=0, \ldots, m-1$,

$$
X_{k}^{\min }=\left\{g \in X_{k-1}^{\min } \mid \delta_{k}(g) \geq \delta_{k}\left(g^{\prime}\right) \text { for all } g^{\prime} \in X^{\min }\right\},
$$

where $X_{-1}^{\min }=X^{\text {min }}$. By construction we have that $X_{k}^{\min } \subseteq X_{k-1}^{\min }, k=$ $0, \ldots, m-1$ and that $X_{m-1}^{\min }$ is not empty. In the following lemmas we show that any network in $X_{m-1}^{\min }$ satisfies a number of properties.

First, observe that in any $g \in X_{m-1}^{\min }$ each group is connected. If not, then there is a group, say group $\ell$, of which two players belong to a different component in $g$. Since $f(0) \leq 1$ and in case of ties sponsoring a link is preferred to not sponsoring, any best reply of a player in $N_{\ell}$ contains an internal link to a player in $N_{\ell}$ that he does not observe in $g$. Moreover, since the network is minimal, if an updating player deletes an internal link, then this link will be replaced by an alternative internal link. Therefore, any update of a player in $N_{\ell}$ leads to a network $g^{\prime}$ with more links of length zero than in $g$, which contradicts that $g \in X_{m-1}^{\min }$. So, any group is connected. Now, the next lemma states that in a network $g \in X_{m-1}^{\min }$ not only each group is connected, but also that each group is either entirely internally connected or entirely externally connected.

Lemma 3. For each $g \in X_{m-1}^{\min }$ it holds that $\mathcal{N}^{I}(g) \cup \mathcal{N}^{E}(g)=\mathcal{N}$, i.e. any group is either entirely internally, or entirely externally connected.

Since the proof is given in Appendix A, here we only illustrate the lemma by an example.

\section{Example 2.}

Consider Figure 7 but with link $(6,2)$ replaced by link $(2,3)$ and suppose that this modified network $g$ belongs to $X_{m-1}^{\min }$ for some RC $X$. In the network $g$ group $N_{1}$ is neither entirely internally connected nor entirely externally. Supposing that $f(1)<2$ any of the players 1, 2, 3 and 6 plays a best reply to $g$. However, when players 2 and 6 update simultaneously, it may happen that player 2 replaces his link to player 3 by a link to player 1 and that at the same time player 6 replaces his link to player 3 by a link to player 2 . For both players this is a best reply to $g$. Let $g^{\prime}$ be the resulting network. Clearly, because of the miscoordination in the previous step, player 3 is now 
isolated of the other players in his group and thus in $g^{\prime}$ neither of the players of group $N_{1}$ plays an optimal reply. Now, let player 3 update. Then this player will sponsor a link to (one of) of the other players in $N_{1}$, say player 1 . When successively player 6 gets the turn to update, this player will delete his link to player 2 , because he is connected already to 2 through the path via 9,8 and 1 . Now, the resulting network is minimal again, but contains one more links of length zero than the original network $g$, which contradicts that $g \in X_{m-1}^{\min }$.

The next lemma excludes that in a network $g \in X_{m-1}^{\min }$ a player observes an other player in his own group through an external link.

Lemma 4. Let $g \in X_{m-1}^{\min }$ and let $i \in N_{\ell}$ and $j \in N_{k}$ for some $k \neq \ell$. If $\{i, j\} \in \bar{g}, N_{k} \in \mathcal{N}^{E}(g)$ and there is a player $h \in N_{k}, h \neq j$, that is observed by $j$ via $i$, then $(i, j) \in g$.

\section{Example 3.}

Consider Figure 8 but with link $(12,8)$ replaced by link $(8,12)$ and suppose that this modified network $g \in X$ for some RC $X$. In this network $g$ we then have that player 8 observes player 7 in his group $N_{3}$ through his link to player 12 in $N_{4}$, i.e. $i=12, j=8, h=7, k=3$ and $\ell=4$ and $(8,12) \in g$. The lemma says that this excludes that $g$ is in $X_{m-1}^{\min }$. Indeed, when player 8 has the possibility to update, he will replace his link to 12 by a link to 7 , increasing the number of links of length zero. Observe that in the original network in Figure 8 it holds that $(12,8) \in g$ and thus satisfies the property stated in the lemma.

We have observed already that any group is connected. The previous lemma implies that when $g \in X_{m-1}^{\min }$, each player of an entirely externally connected group observes the other members of his group via links he receives. Thus if every player belongs to an entirely externally connected group, there are at least as many links as players, which is impossible in a minimal network. So, at least one group is not entirely externally connected. Together with Lemma 3 we obtain without further proof the following result.

Lemma 5. For any $g \in X_{m-1}^{\min }$ it holds that $\mathcal{N}^{I}(g) \neq \emptyset$, i.e. there is at least one group that is entirely internally connected.

The next lemma says that any player on a path in $g$ between two players $j$ and $j^{\prime}$ of an entirely externally connected group does not have any best reply in which $j$ and $j^{\prime}$ become disconnected. Recall that there are no internal links in an entirely externally connected group.

Lemma 6. Let $g \in X_{m-1}^{\min }$ and let players $j$ and $j^{\prime}$ be two distinct players in some group $N_{\ell} \in \mathcal{N}^{E}(g)$. Then if $i$ is on the path between $j$ and $j^{\prime}$, then there is a path between $j$ and $j^{\prime}$ in any network $g^{\prime}=g_{-i} \cup b_{i}(g)$ for all $b_{i}(g) \in B_{i}(g)$. 


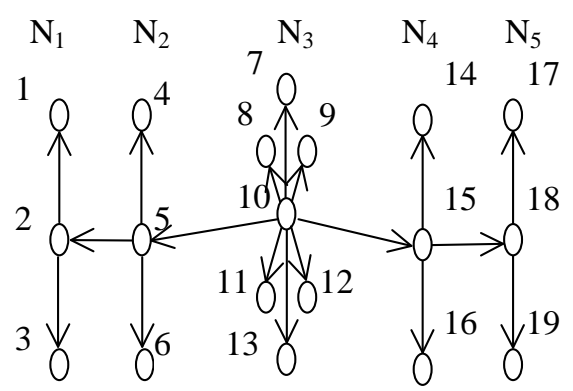

Figure 10. Network in an STCS for $f(1) \leq 3$.

\section{Example 4.}

Suppose that the network $g$ given in Figure 7 belongs to $X$ for some RC $X$ and assume that $f(2)>1$. Then the players 1 and 2 of the entirely externally connected group $N_{1}$ become unconnected when player 8 updates. Indeed this player will delete his link to player 1 . Next, let player 2 update. Then this player will sponsor an additional link to 1 . The resulting network is minimal and contains more internal links then g, excluding that $g \in X_{m-1}^{\min }$.

The next lemma states that in any $g \in X_{m-1}^{\min }$ each pair of players observes each other.

Lemma 7. Any network $g$ in $X_{m-1}^{\min }$ is connected.

\section{Example 5.}

Suppose that the network given in Figure 10 belongs to $X_{m-1}^{\min }$ for some RC $X$ and assume that $3<f(1)<7=\max _{\ell}\left|N_{\ell}\right|=\left|N_{3}\right|$. Denote the network by $g^{0}$. When player 5 updates the network becomes $g^{1}=g^{0} \backslash\{(5,2)\}$, in which player 5 has deleted the link $(5,2)$ because $f(1)>3$. Next, let player 10 update. Then this player deletes the link $(10,5)$ and we obtain $g^{2}=g^{1} \backslash\{(10,5)\}$. However, next let player 5 update again. Then this player will add the link $(5,10)$ since $f(1)<7$ and we obtain the network $g^{3}=g^{2} \cup\{(5,10)\}$. Finally, let some player $i \in N_{1}$ update. Then this player will add a link $(i, j)$ to some player $j \in N_{2}$ and we obtain the network $g^{4}=g^{3} \cup\{(i, j)\}$. Since $g^{0} \in X$ and it is supposed that $X$ is RC, all networks $g^{i} \in X, i=1, \ldots, 4$. Moreover all networks have the same number of internal links, but only the connected networks $g^{0}$ and $g^{4}$ contain four links of length one. The other three unconnected networks have only two or three links of lengths one and therefore they do not belong to $X_{m-1}^{\min }$.

The next lemma shows that for any $g \in X_{m-1}^{\min }$ all links of length two or more are stable, i.e. each player $i$ has a best reply $b_{i}(g) \in B_{i}(g)$ such that $b_{i}(g)$ contains all links $(i, j)$ in $g_{i}$ of length two or longer. 
Lemma 8. Suppose $g \in X_{m-1}^{\min }$. Then for any $i$ it holds that

$$
\left\{(i, j) \in g_{i} \mid \text { length }(i, j) \geq 2\right\} \subseteq b_{i}(g)
$$

for some $b_{i}(g) \in B_{i}(g)$.

\section{Example 6.}

We show two examples contradicting that $g \in X_{m-1}^{\min }$ when a player prefers to delete or replace a link of length two. First, consider Figure 10 but with link $(15,18)$ replaced by $(9,18)$ and suppose that this modified network $g^{0} \in X$ for some RC $X$. Further assume that $3<f(1)<7$. In this case, player 9 strictly prefers to delete the link $(9,18)$ of length 2 . So, when 9 updates, we get $g^{1}=g^{0} \backslash\{(9,18)\}$. However, when succeedingly player 18 updates, we obtain the network $g^{2}=g^{0} \cup\{(18, j)\}$ for some $j \in N_{4}$. Since this network contains one more link of length one than network $g^{0}$, it follows that $g^{0}$ is not in $X_{m-1}^{\min }$.

Next, consider Figure 10 but with link $(10,15)$ replaced by $(10,17)$ and suppose that this modified network $g^{0} \in X$ for some RC $X$. Clearly, when player 10 updates, he prefers to replace $(10,17)$ by a link to group 4 . Again, the resulting network is minimal and has one more link of length one than $g^{0}$, contradicting that $g^{0} \in X_{m-1}^{\min }$.

We are now ready to show that each $\mathrm{RC} X$ contains a non-empty subset of Nash networks. Therefore we define the subset of networks of $X_{m-1}^{\min }$, denoted by $\widehat{X}_{m-1}^{\min }$, satisfying that all links of length one stable, i.e.

$$
\begin{aligned}
\widehat{X}_{m-1}^{\min }=\left\{g \in X_{m-1}^{\min } \quad \mid\right. & \text { for any } i \text { there exists } b_{i}(g) \\
& \text { such that } \left.\left\{(i, j) \in g_{i} \mid \text { length }(i, j)=1\right\} \subseteq b_{i}(g)\right\} .
\end{aligned}
$$

From Lemma 8 we already know that in any $g \in X_{m-1}^{\min }$ any link of at least length two is stable. Moreover, all internal links (of length zero) are stable, since any $g \in X_{m-1}^{\min }$ is minimal and $f(0) \leq 1$. So, for a network $g \in \widehat{X}_{m-1}^{\min }$ it holds that all links are stable, i.e. for any $i$ it holds that there is a best reply $b_{i}(g)$ such that $(i, j) \in b_{i}(g)$ for any link $(i, j) \in g_{i}$. So, for some given player $i$, referring to the notation used in Lemma 1, we have that any player $j$ to which player $i$ sponsors a link in the network $g$ belongs to the set $D_{h}$ of some component $C_{h}$ (not containing $i$ ) in $g_{-i}$, and moreover that in $g$ any player $i$ sponsors a link to at most one player in any component $C_{h}$. Moreover, it should be observed that $D_{h} \neq \emptyset$ for any $C_{h}$ not containing $i$, because $g$ is connected. So, without further proof, it follows now immediately from Lemma 1 that each network in $\widehat{X}_{m-1}^{\min }$ is a Nash network.

Lemma 9. Any network $g \in \widehat{X}_{m-1}^{\min }$ is a Nash network, i.e.

$$
\widehat{X}_{m-1}^{\min }=\left\{g \in X_{m-1}^{\min } \mid g \in B(g)\right\} .
$$

The next lemma is crucial for the proof of Theorem 5 and states that $\widehat{X}_{m-1}^{\min }$ is not empty, implying that any MCS contains at least one Nash network. 
Lemma 10. Let $X$ be an $R C$. Then the set $\widehat{X}_{m-1}^{\min }$ of Nash networks is not empty.

\section{Example 7.}

Suppose that the network $g$ given in Figure 10 belongs to $X_{m-1}^{\min }$ for some RC $X$ and assume that $6<f(1)<7$. Clearly, $g$ is not Nash. When player 10 updates, he deletes both $(10,5)$ and $(10,15)$ because he only observes 6 players through each of these links. Next, when player 5 updates, he deletes $(5,2)$, but he will add a link to group 3 . Then let any one player of group 1 update: he creates a link to group 2 . Similarly, let respectively player 15 and a player from group 5 update. This process leads to a Nash network $g^{\prime}$. Since $\delta_{k}\left(g^{\prime}\right)=\delta_{k}(g)$ for any length $k$ and it is supposed that $g \in X_{m-1}^{\min }$, it follows that $g^{\prime} \in \widehat{X}_{m-1}^{\min }$, showing that the latter set is not empty.

We now continue with some properties for networks $g$ in $\widehat{X}_{m-1}^{\min }$. The first property says that when $g \in \widehat{X}_{m-1}^{\min }$ each player $i$ sponsors in any $g^{\prime} \in B(g)$ the same number of links as in $g$ to each group.

Lemma 11. Let $g$ be a network in $\widehat{X}_{m-1}^{\min }$ and $g^{\prime}$ a network in $B(g)$. Then for each $i$ and each $\ell$ it holds that

\section{Example 8.}

$$
\left|\left\{(i, j) \in g \mid j \in N_{\ell}\right\}\right|=\left|\left\{(i, j) \in g^{\prime} \mid j \in N_{\ell}\right\}\right|
$$

Consider Figure 8 , but with link $(5,9)$ replaced by $(1,7)$ and suppose that the modified network $g$ is in $\widehat{X}_{m-1}^{\min }$ for some RC $X$. In this network player 5 is indifferent between the link $(5,1)$ and a link to any one member of group 3 (or to any other member of group 1). So, the lemma does not hold, because player 5 can replace the link $(5,1)$ by for instance the link $(5,8)$. However, when player 5 is doing so, then player 1 strictly prefers to replace $(1,7)$ by a link to group 2. This violates Lemma 8 , showing that $g$ is not in $X_{m-1}^{\min }$ and thus also not in $\widehat{X}_{m-1}^{\min }$.

Lemma 11 implies the following corollary:

Corollary 1. Let $g$ be a network in $\widehat{X}_{m-1}^{\min }$ and $(i, j) \in g, i \in N_{\ell}, j \in N_{k}$ where $k \neq \ell$. Then $i$ is not indifferent between a link to $j$ and a link to some $j^{\prime} \notin N_{k}$.

Proof We prove this by contradiction. Let $i$ update. Because $g$ is a Nash network, by Lemma $1 i$ may select $b_{i}(g)=\left(g_{i} \backslash\{(i, j)\}\right) \cup\left\{\left(i, j^{\prime}\right)\right\}$. However, since $j^{\prime} \notin N_{k}$ player $i$ then sponsors less links to group $k$ then before. This is a contradiction with Lemma 11.

The next property says that when in a Nash network of an RC a player in an entirely externally connected group observes another player in his group through a link sponsored to him by a player $i$, then he observes all players in his group through the same link. Observe that player $i$ does not belong to the same group, otherwise the group is not entirely externally connected. 
Lemma 12. Let $g \in \widehat{X}_{m-1}^{\min }$ and $j$ and $j^{\prime}$ two distinct players in some group $N_{\ell} \in \mathcal{N}^{E}(g)$. When $(i, j) \in g$ and $i$ is on the path between $j$ to $j^{\prime}$, then $i$ is on the path between $j$ and $h$ for all $h \in N_{\ell} \backslash\{j\}$.

Example 9. Consider Figure 8 but with link $(12,8)$ replaced by link $(5,8)$ and suppose that this modified network $g \in X$ for some RC $X$. Now, player 9 in the entirely externally connected group $N_{3}$ observes player 8 through the link sponsored by player 5 , but he observes player 10 through the link sponsored by 12 . Hence the lemma is not satisfied. Indeed, observe that in $g$ player 12 is indifferent between a link to 8 and a link to 9 , while player 5 is indifferent between a link to 9 and a link to 10 . Let these players update simultaneously, such that 5 switched to 10 and 12 switched to 8 . Then 9 becomes isolated. Next, when 9 updates, he will sponsor an internal link, for instance to 10. Finally, when 5 updates again, he will delete one of his links to 8 and 10. The resulting network is minimal, but has more internal links than $g$. Hence $g$ is not in $X_{m-1}^{\min }$ and thus also not in $\widehat{X}_{m-1}^{\min }$.

Next, we define a subset of the set of Nash networks $\widehat{X}_{m-1}^{\min }$, to be denoted by $\widetilde{X}_{m-1}^{\min }$, satisfying that players within a entirely internally connected group in the network are organized in a center-sponsored star (CSS), i.e.

$$
\begin{aligned}
(4.1) \widetilde{X}_{m-1}^{\min }=\left\{g \in \widehat{X}_{m-1}^{\min } \quad \mid\right. & \text { for all } N_{\ell} \in \mathcal{N}^{I}(g), \text { there exists } i \in N_{\ell} \\
& \text { such that } \left.\left(i, i^{\prime}\right) \in g \text { for each } i^{\prime} \in N_{\ell} \backslash\{i\}\right\} .
\end{aligned}
$$

Bala and Goyal (2000) showed that in a model without cost heterogeneity players are organized in a CSS. Because in our model there is no cost heterogeneity within groups and all paths connecting players of entirely internally connected groups consist only of internal links, we expect that their result extends to entirely internally connected groups. Indeed we will show in Section 5 that in any $g$ in a STCS each entirely internally connected group is CSS. As a preliminary result, the next lemma says that for any MCS $X$, the subset $\widetilde{X}_{m-1}^{\mathrm{min}}$ wherein the architectural structure of any internally connected group is a central-sponsored star (CSS) is not empty. For the proof we refer again to Appendix A.

Lemma 13. Let $X$ be an $R C$. Then $\widetilde{X}_{m-1}^{\min }$ is not empty.

The final lemma shows that within an RC $X$ the set of best replies is the same for any $g \in X$ by showing that for any $g \in X$ the best reply set is the same as the best reply set for a given network $g^{*} \in \widetilde{X}_{m-1}^{\min }$.

Lemma 14. Let $g^{*} \in \widetilde{X}_{m-1}^{\min }$. Then then $B(g)=B\left(g^{*}\right)$ for any $g \in X$.

Again, the proof of the lemma is given in Appendix A. Without further ado we can present the final lemma.

Lemma 15. Let $X \subseteq \mathcal{G}$ be an $R C$, and let $g \in X$. Then $B(g)=X$. 
Proof By lemma 14 it is sufficient to show that every network in an RC is a Nash network. Suppose that $g \in X$ is not a Nash network, and consider some $g^{0} \in \widetilde{X}_{m-1}^{\min }$. Because both networks belong to the same RC, there is a path of networks from $g^{0}$ to $g$. The dynamics imply (see Equation 2.2) that for each player either $g_{i}=g_{i}^{0}$ or $g_{i} \in B_{i}\left(g^{\prime}\right)$ for some $g^{\prime}$ on the path of networks from $g^{0}$ to $g$. Because $g^{0}$ is a Nash network, $g_{i}^{0} \in B_{i}\left(g^{0}\right)$ for all $i \in N$. Hence for all $i \in N$, there is a network $g^{\prime} \in X$ such that $g_{i} \in B_{i}\left(g^{\prime}\right)$. By lemma 14, it follows that $g_{i} \in B_{i}(g)$ for all $i \in N$. Therefore $g \in B(g)$ for all $g \in X$.

Thus we find that any RC $X$ has the feature that $B(g)=X$. Hence $X$ super tight curb set, as is stated in Theorem 5 .

Proof Theorem 5 The proof follows from the Lemmas 2-14.

Because an STCS is an MCS, it implies that every RC is an MCS. Moreover, because any MCS contains an RC, the Lemma implies that any MCS is an RC. Without further proof we can therefore state the following corollary.

Corollary 2. Let be given a network formation game with $m$ groups of players $N_{1}, \ldots, N_{m}, m \geq 2$ and $\left|N_{\ell}\right| \geq 2$ for all $\ell$, and with a strictly increasing cost function $f(\cdot)$ satisfying $f(0) \leq 1$ and $f(1) \leq \max _{\ell}\left|N_{\ell}\right|$. If $X \subseteq \mathcal{G}$ is an MCS, then $X$ is also an STCS and an RC of the dynamics specified in Equation 2.1.

With this corollary, we can characterize the networks in an MCS of the MG model.

\section{Characterization of Networks in Minimal Curb Sets}

In the previous section we have seen that in the Multiple Group network formation game any RC contains an STCS, and therefore that every MCS is super tight. Note that the characterization of the MCS networks in this model is possible only because of Theorem 5 . Without that result we can not establish any non-trivial a priori requirement on the networks in the game that holds to be true for all networks contained in an MCS. For example, we can not even exclude that non-Nash networks are part of some MCS (for example in the MCS $Y$ of the game in Figure 5). The same problems apply to a subset of the networks in an MCS: the recurrent class.

In contrast, the concept of an STCS is much more restrictive. It demands for instance that all networks in that set are Nash equilibria. Therefore the result that every MCS is super tight is crucial in the characterization of the MCS networks.

In this section we assume again that $f(0) \leq 1$, and $f(1) \leq \max _{\ell}\left|N_{\ell}\right|$. The characterization follows in three steps. First we show in the Theorems 7, 8 and 9 that any network in an MCS satisfies seven properties. In our proofs we use the result that any MCS is also an RC from the dynamics 
specified in Equation 2.1. The five properties given in Theorem 7 follow straightforwardly from the next theorem below, that states that any MCS $X$ is equal to its subset $\widetilde{X}_{m-1}^{\min }$ as specified in Equation ??. The Theorems 8 and 9 state two additional properties. Finally Theorem 10 shows that these seven properties are also sufficient to guarantee that a network is part of an MCS.

Theorem 6. Let $X \subseteq G$ be an MCS. Then $X=\widetilde{X}_{m-1}^{\min }$.

Proof By Lemma 13 we have that $\widetilde{X}_{m-1}^{\min } \neq \emptyset$. Let $g^{*}$ be a network in $\widetilde{X}_{m-1}^{\min }$. From Lemma 14 we know that $B(g)=B\left(g^{*}\right)=X$ for all $g \in X$. From this it follows that $X=X_{m-1}^{\min }$. Suppose not. Then there exists a network $g \in B\left(g^{*}\right) \backslash X_{m-1}^{\min }$. So, there must exist a player $j$ who in $g$ either sponsors more links than he does in $g^{*}$, or has deleted a link, or has replaced a link by a longer one. However, each of these possibilities contradicts that $g_{j} \in B_{j}\left(g^{*}\right)$.

Since $g \in X=B(g)$ for any $g \in X$, it also follows that any $g \in X$ is a Nash network, thus $X=\widehat{X}_{m-1}^{\min }$. Finally from $g \in B\left(g^{*}\right)$ and Lemma 11 it follows that the set of internal links in $g$ is equal to the set of internal links in $g^{*}$. So a group in $g$ is in $\mathcal{N}^{I}(g)$ and CSS if and only if it is in $\mathcal{N}^{I}\left(g^{*}\right)$ and CSS. Hence $g \in \widetilde{X}_{m-1}^{\min }$.

Theorem 7. Let $X \subseteq \mathcal{G}$ be an $M C S$. Then each network $g \in X$ satisfies the following properties:

A. $g$ is a Nash network and is minimal connected,

B. $\mathcal{N}^{I}(g) \cup \mathcal{N}^{E}(g)=\mathcal{N}$ and $\mathcal{N}^{I}(g) \neq \emptyset$,

C. if $N_{\ell} \in \mathcal{N}^{I}(g)$ then $N_{\ell}$ is a CSS,

D. Let $(i, j) \in g$, where $j \in N_{\ell}$. Then for each $g^{\prime} \in B_{i}(g)$

$$
\left|\left\{(i, j) \in g \mid j \in N_{\ell}\right\}\right|=\left|\left\{(i, j) \in g^{\prime} \mid j \in N_{\ell}\right\}\right|,
$$

E. Let $(i, j) \in g, i \in N_{\ell}, j \in N_{k} \in \mathcal{N}^{E}(g)$. If $j$ observes any player $j^{\prime} \in N_{k} \backslash\{j\}$ via $i$, then all $j^{\prime \prime} \in N_{k} \backslash\{j\}$ are observed by $j$ via $i$.

Proof By Theorem 6 we have that any $g \in X$ is in $\widetilde{X}_{m-1}^{\text {min }}$, which shows the properties $\mathbf{A}$ and $\mathbf{C}$. Further, any $g$ satisfies all the properties given in the lemmas of Section 4. So, property B follows from Lemma 3 and Lemma 5, property $\mathbf{D}$ from Lemma 11 and property $\mathbf{E}$ from Lemma 12.

The five properties of Theorem 7 do not by themselves prevent the three causes of changes in best reply sets: (i) miscoordination, (ii) a too large decrease of the number of people exclusively observed by some player $i$ through some link $\left(i, i^{\prime}\right)$ and (iii) the entrance of some player $j$ in the set of 
people exclusively observed by some player $i$ through some link $\left(i, i^{\prime}\right)$, where $i$ is not further from $j$ than from $i^{\prime}$. In Theorems 8 and 9 below we show that any network in an MCS satisfies two additional properties that prevent these three things to happen. To do so, recall from Section 2.3 that for a network $g \in \mathcal{G}$, player $i \in N$ and link $\left(i, i^{\prime}\right), A_{i i^{\prime}}(g)$ is the set of players that $i$ observes in $g$ and that $i$ would not observe if $i$ deletes his link to $i^{\prime}$; that $A_{i i^{\prime}}^{E}(g)$ is the subset of players in $A_{i i^{\prime}}(g)$ that belong to entirely externally connected group containing another player in the same group who is not in $A_{i i^{\prime}}(g)$; and that $D_{i i^{\prime}}^{E}(g)$ is the set of players in entirely externally connected groups such that other players of that group are observed by $i$ via $\left(i, i^{\prime}\right)$ but they themselves are not.

Theorem 8. Let $X \subseteq \mathcal{G}$ be an $M C S$. Then each network $g \in X$ satisfies the following property:

F. Let $\left(i, i^{\prime}\right),\left(j, j^{\prime}\right) \in g$ be two links, $i \in N_{\ell}$ and $i^{\prime} \in N_{\ell^{\prime}}$ with $\ell \neq \ell^{\prime}, j \in N_{k}$ and $j^{\prime} \in N_{k^{\prime}}$ with $k \neq k^{\prime}$, such that $i \in A_{j j^{\prime}}(g)$ and $j \in A_{i i^{\prime}}(g)$. Then: if $j^{\prime} \in A_{i i^{\prime}}^{E}(g)$ then $i^{\prime} \notin A_{j j^{\prime}}^{E}(g)$.

Proof Suppose $\mathbf{F}$ is violated. Then it is easy to see that by letting $i$ and $j$ update simultaneously we can get the following network

$$
g^{\prime}=\left(g \backslash\left\{\left(i, i^{\prime}\right),\left(j, j^{\prime}\right)\right\}\right) \cup\left\{\left(i, i^{\prime \prime}\right),\left(j, j^{\prime \prime}\right)\right\}
$$

with $i^{\prime \prime} \in D_{j j^{\prime}}^{E}(g) \cap N_{\ell^{\prime}}$ and $j^{\prime \prime} \in D_{i i^{\prime}}^{E}(g) \cap N_{k^{\prime}}$. Obviously $g^{\prime}$ is not connected, which implies by property $\mathbf{A}$ that $g^{\prime} \notin X$ which contradicts that $X$ is MCS.

Property $\mathbf{F}$ says that miscoordination can not occur when two players update simultaneously their strategy in a network $g$ that belongs to an MCS $X$. For an example violating this property, see Figure 11. For this graph we have that $A_{2,5}(g)=\{4, \ldots, 14,16,17,18\}, A_{17,14}(g)=\{1,2,3,5, \ldots, 15\}$ with $A_{2,5}^{E}(g)=\{13,14\}, A_{17,14}^{E}(g)=\{5,6\}$ and $D_{2,5}^{E}(g)=\{15\}, D_{17,14}^{E}(g)=$ $\{4\}$, so that property $\mathbf{F}$ is violated. Now, players 2 and 17 miscoordinate when 2 replaces his link to 5 by a link to 4 and when 17 replaces his link to 14 by a link to 15 , resulting in a unconnected network. Property $\mathbf{F}$ prevents from such a miscoordination.

To state the next theorem, we define for an MCS $X$ and for each link $\left(i, i^{\prime}\right), i$ and $i^{\prime}$ in different groups, contained in at least one $g \in X$ the set

$$
A_{i i^{\prime}}^{\min }(X)=\cap_{\left\{g \in X \mid\left(i, i^{\prime}\right) \in g\right\}} A_{i i^{\prime}}(g)
$$

as the set of players that $i$ observes exclusively through his link to $i^{\prime}$ in each network in $X$ containing this link and the set

$$
A_{i i^{\prime}}^{\max }(X)=\cup_{\left\{g \in X \mid\left(i, i^{\prime}\right) \in g\right\}} A_{i i^{\prime}}(g)
$$

as the set of players that $i$ observes exclusively through his link to $i^{\prime}$ in at least one network containing this link. In Appendix B we prove the next 


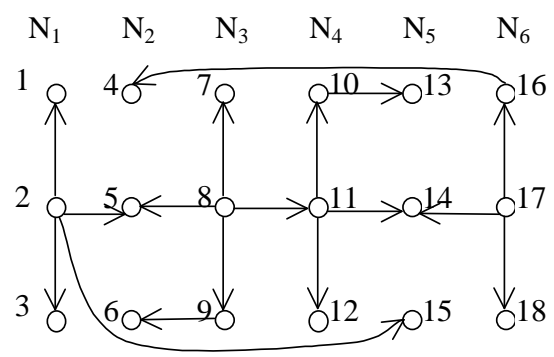

Figure 11. Miscoordination possible between players 2 and 17.

lemma, in which we define $A_{i i^{\prime}}^{\min }(X)=A_{i i^{\prime}}^{\max }(X)=\emptyset$ if there does not exist a network $g \in X$ containing the link $\left(i, i^{\prime}\right)$.

Lemma 16. Let $X \subseteq \mathcal{G}$ be an MCS. Then for any pair of players $i$ and $i^{\prime}$ in different groups there exist networks $g^{\min }$ and $g^{\max }$ in $X$ such that

$$
A_{i i^{\prime}}^{\min }(X)=A_{i i^{\prime}}\left(g^{\min }\right)
$$

and

$$
A_{i i^{\prime}}^{\max }(X)=A_{i i^{\prime}}\left(g^{\max }\right) .
$$

According to this lemma there exist networks such that the set of players exclusively observed by $i$ through his link to an $i^{\prime}$ in some other group is precisely equal to the set of players that he observes exclusively through this link in each network that contains the link, respectively in at least one network that contains the link. This implies the next corollary.

Corollary 3. Let $X \subseteq \mathcal{G}$ be an MCS and $i$ and $i^{\prime}$ two players in different groups. Then for any $g \in X$ such that $\left(i, i^{\prime}\right) \in g$ it holds that

$$
A_{i i^{\prime}}\left(g^{\min }\right) \subseteq A_{i i^{\prime}}(g) \subseteq A_{i i^{\prime}}\left(g^{\max }\right) .
$$

We are now ready to state the next theorem.

Theorem 9. Let $X \subseteq \mathcal{G}$ be an MCS. Then for each network $g \in X$ and link $\left(i, i^{\prime}\right)$ in $g$ with $i \in N_{\ell}$ and $i^{\prime} \in N_{\ell^{\prime}}, \ell \neq \ell^{\prime}$, it holds that:

G.1. $\left|A_{i i^{\prime}}^{\min }(X)\right| \geq f\left(\left|\ell^{\prime}-\ell\right|\right)$.

G.2. for all $\ell^{\prime \prime} \neq \ell^{\prime}$ such that $\left|\ell^{\prime \prime}-\ell\right| \leq\left|\ell^{\prime}-\ell\right|$, there does not exist $j^{\prime \prime} \in A_{i i^{\prime}}^{\max }(X) \cap N_{\ell^{\prime \prime}}$.

G.3. $A_{i i^{\prime}}^{\max }(X) \cap N_{\ell^{\prime}}=A_{i i^{\prime}}^{\min }(X) \cap N_{\ell^{\prime}}$.

Proof Suppose that G.1 is violated, i.e. $\left|A_{i i^{\prime}}^{\min }(X)\right|<f\left(\left|\ell^{\prime}-\ell\right|\right.$ ) (see for example see Figure 12). By Lemma 16 there exists a network $g^{\text {min }} \in X$ such that $A_{i i^{\prime}}\left(g^{\mathrm{min}}\right)=A_{i i^{\prime}}^{\min }(X)$. However, this contradicts that $g^{\mathrm{min}}$ is a Nash equilibrium. 


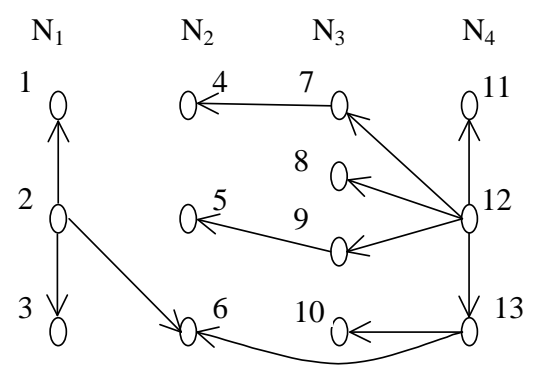

FiguRE 12. Nash network for $f(2) \leq 4$. It belongs to an MCS only if $f(2) \leq 1$.

Next, suppose that G.2 is violated. Again, by Lemma 16 there is a network $g^{\max } \in X$ such that $A_{i i^{\prime}}\left(g^{\max }\right)=A_{i i^{\prime}}^{\max }(X)$. If there exist $N_{\ell^{\prime \prime}} \neq N_{\ell^{\prime}}$ and $j^{\prime \prime} \in A_{i i^{\prime}}^{\max }(X) \cap N_{\ell^{\prime \prime}}$ such that $\left|\ell^{\prime \prime}-\ell\right| \leq\left|\ell^{\prime}-\ell\right|$, property $\mathbf{D}$ is violated.

Finally, suppose that G.3. is violated. Note that in any network $g^{\prime} \in X$, player $i$ is indifferent between any pair of players in $A_{i i^{\prime}}\left(g^{\prime}\right) \cap N_{\ell^{\prime}}$. However, he is not indifferent between such a player and a player in $N_{\ell^{\prime}} \backslash A_{i i^{\prime}}^{\max }(X)$. Therefore $A_{i i^{\prime}}^{\max }(X) \cap N_{\ell^{\prime}} \neq A_{i i^{\prime}}^{\min }(X) \cap N_{\ell^{\prime}}$ implies $B\left(g^{\min }\right) \neq B\left(g^{\max }\right)$. This constitutes a contradiction with $g \in X$.

The three properties of $\mathrm{G}$ deal with the set of players that a sponsor of a link observes through that link. Clearly this is relevant for the set of best replies of the sponsor. And the set of players observed through that link may depend on the strategies of other players, even within an MCS. One of the things that may happen is that the size of this set becomes smaller. If it becomes too small, the sponsor, who previously preferred to sponsor this link, now prefers to delete it. Look for instance at the network in figure 12 . It is a Nash network if $f(2) \leq 4$. However, unless $f(2) \leq 1$, the network is not part of an MCS because player 2 might replace his link to 6 by a link to 5. G.1. says that this cannot occur within an MCS.

Similarly, the set of players observed through a link by its sponsor may grow. It may be the case that a player enters this set that is not further from the sponsor than the current recipient of the link. For example consider Figure 11 but with $(17,14)$ replaced by $(14,17))$, In this network, player 4 is the closest player to 16 in the set $A_{16,4}(g)$. However, if player 2 replaces $(2,5)$ by $(2,4)$, then player 16 will also observe player 15 through his link $(16,4)$, and player 16 will now have a best reply in which he sponsors a link to 15. More generally: the set of players observed through a link by its sponsor that are closest to the sponsor is not allowed to change within an MCS. This is ensured by G.2. and G.3.

Above we have shown that any network in an MCS satisfies the properties $\mathbf{A}-\mathbf{G}$, i.e. the properties A-G are necessarily satisfied by any network in an 
MCS. The next theorem says that these properties are also sufficient to be a network in an MCS.

Theorem 10. If a network $g$ satisfies properties $\boldsymbol{A}-\boldsymbol{E}$ and $\boldsymbol{G}^{15}$, and all networks $g^{\prime} \in B(g)$ satisfy property $\boldsymbol{F}$, then there is an MCS X containing $g$.

Proof The theorem holds when (i) $g$ is a Nash network, (ii) there is no sequence of best replies possible such that the set of best replies for any player, say $i$, is changed, and (iii) each network that is the result of the union of best replies of the players against the current network $g$ can be reached by a series of best replies.

The first condition follows because of property A. Also the third condition follows immediately. Just, let every player update at the same time and let them select the corresponding best reply. It remains to consider the the second condition.

Suppose that $g$ satisfies the properties A-G but is not element of an MCS. Then there are three ways in which a series of best replies can lead to a change in the set of best replies of some player. The first way is that the set of players observed through a link becomes too small for the sponsor compared to the cost of that link. This is impossible without violating G.1. The second possibility is that the set of players observed through a link expands in such a way that a 'new' player in that set is at least as close to the sponsor as the recipient of the link. This would violate either G.2. or G.3. Alternatively a player in this set that is one of the players that are closest to the sponsor of the link may leave the set. In that case the sponsor would have one alternative less for his link. This however would violate G.3. The third way is that two or more players update simultaneously so that one or more cycles are created. This would violate F. To see why, consider a minimal subset of these players denoted by $N_{\text {cycle }}=\left\{i_{1}, \ldots, i_{k}\right\}$ such that one cycle is created. Denote the strategies for which this cycle is created by $b_{i_{j}}$ for player $i_{j}, j \in\{1, \ldots, k\}$. Let players $i_{3}$ to $i_{k}$ update simultaneously with strategies $b_{i_{3}}$ to $b_{i_{k}}$ respectively. Call the resulting network $g^{\prime}$. Network $g^{\prime}$ is minimal by construction (minimality of $N_{\text {cycle }}$ ) and connected, because $g$ was a Nash network (therefore the number of links has not decreased). By construction, if $i_{1}$ and $i_{2}$ now select $b_{i_{1}}$ respectively $b_{i_{2}}$, then miscoordination occurs. Therefore there exist some external links $\left(i_{1}, j_{1}\right),\left(i_{2}, j_{2}\right) \in g^{\prime}$, such that $i_{1} \in A_{i_{2} j_{2}}^{E}$ and $i_{2} \in A_{i_{1} j_{1}}^{E}$. This is a contradiction because $g^{\prime} \in B(g)$ and $g^{\prime}$ does not satisfy property F. Hence $B\left(g^{\prime}\right)=B(g)$ for all $g^{\prime} \in B(g)$, and thus $g$ is part of an STCS.

\footnotetext{
${ }^{15}$ With in property $\mathbf{G} A_{i i^{\prime}}^{h}(X)$ defined to be equal to $A_{i i^{\prime}}^{h}(g), h \in\{\min , \max \}$ (see appendix B for the definition of $\left.A_{i i^{\prime}}^{h}(g)\right)$.
} 


\section{TYPES OF NETWORKS IN AN MCS}

In the previous section we have fully characterized the networks that may arise in an MCS of the multiple group network formation game. A network $g$ is in an MCS if and only if $g$ satisfies the properties A-G. Moreover, we also have that any MCS is an STCS. So, in particular we have that any $g$ in an MCS $X$ is a Nash network and that $B(g)=X$ for any $g \in X$. In this section we want to consider several types of networks that may arise in an MCS.

When $f(k) \leq 1$ for all $k$, then any network $g$ is which one of the players sponsors a link to each other player is a strict Nash equilibrium. Hence, when the costs are small enough, we obtain again the result of Bala and Goyal (2000): any CSS is a strict Nash network and therefore also an MCS with this CSS as its single element. Clearly, in the model with multiple groups a CSS is not Nash anymore when $f(1)>1$. In the sequel we assume again that $f(0) \leq 1, f(1) \leq \max _{\ell}\left|N_{\ell}\right|$, that $f$ is strictly increasing. We consider which types of networks may arise under these assumption. For clarifying reasons, we mainly restrict ourselves to the case $m=3$. For this case we distinguish three types of MCS-networks.

With the description of each type, a figure is depicted with schematic drawings of the different possible MCSs within that type. These schematic illustrations abstract from the players. Therefore, internal links are not represented. Instead an entirely internally connected group is represented by an 'I' and an entirely externally connected group is represented by an 'E'. An arrow between two groups, means that there is at least one link between the players in these groups. The arrow will be dashed if the recipient group is partitioned into more than one component when any of these links are deleted.

Type I networks: these are networks $g$ in which any group is entirely internally connected, i.e. $\left|\mathcal{N}^{I}(g)\right|=m=3$. According to property $\mathbf{C}$, any entirely internally connected group is a CSS. So, in a type I network any group is organized in a CSS and there are just two external links, either both of length 1 or one link of length 1 and one link of length 2 . Therefore we will call networks of this type connected CSS networks. If both external links are of length 1 it follows that there is a link between group 1 and group 2 and a link between group 2 and group 3 . If $f(1)$ is small enough, each of these two links may go in either direction, i.e. from group 1 (respectively 3 ) to group 2 or from group 2 to group 1 (3). So, this gives three possible structures, namely (i) both links go to group 2, (ii) both links go from group 2 to the two other groups and (iii) one link goes from group 1 (or 3) to group 2 and the other from group 2 to 3 (respectively 1 ). For high values of $f(1)$, some of these cases can not occur. For instance, when $f(1)>\left|N_{1}\right|$, then the link between group 1 and 2 must go from 1 to 2 and when $f(1)>\left|N_{1}\right|+\left|N_{2}\right|$, then also the link between groups 2 and 3 must go from 2 to 3 . Further, 


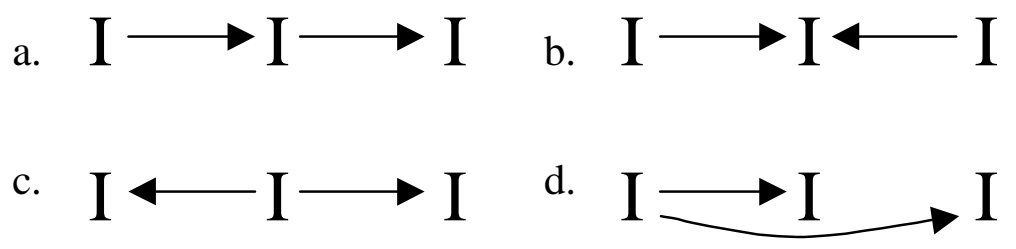

Figure 13. Type I networks

let $i_{\ell}$ be the central player in group $\ell, \ell=1,2,3$, and let $i, j$ be the two players sponsoring the external links, i.e. $i \in N_{\ell}$ and $j \in N_{\ell^{\prime}}$ sponsor links to group $k \neq \ell$, respectively $k^{\prime} \neq \ell^{\prime}$ (of course $i=j$ and $\ell=\ell^{\prime}$ or $k=k^{\prime}$ is possible). Then the unique MCS $X$ containing this network is given by the set of connected CSSs in which $i_{\ell}$ is the central player in group $\ell, \ell=1,2,3$, $i$ sponsors a link to a player in $N_{k}$ and $j$ sponsors a link to a player in $N_{k^{\prime}}$.

In case of a connected CSS with one external link of length 1 and one external link of length 2, w.l.o.g. we may assume that the links are between group 1 and 2 and between group 1 and 3. Then the link of length 1 goes from group 1 to group 2. Otherwise, the player in group 2 sponsoring the link is indifferent between sponsoring a link to group 1 or to group 3, contradicting property D. Further, the link of length 2 goes from group 1 to group 3. Otherwise, the sponsor in group 3 would strictly prefer to replace his link to group 1 by a link to group 2, which contradicts that the network is Nash (property A). So, in this case both links are sponsored by players $i_{1}$ and $i_{2}$ (with possibly $i_{1}=i_{2}$ ) in group 1 , so that this case contains only one structure. Further, let $i_{\ell}$ be the central player in group $\ell, \ell=1,2,3$, and let $j_{1}$ and $j_{2}$ be the two players in $N_{1}$ sponsoring the external links of length 1 , respectively length $2\left(j_{1}=j_{2}\right.$ is possible). Then the unique MCS $X$ containing this network is given by the set of connected CSSs in which $i_{\ell}$ is the central player in group $\ell, \ell=1,2,3$ and $j_{1}\left(j_{2}\right)$ sponsors a link to a player in $N_{2}\left(N_{3}\right)$.

Summing up we have that in case a network is of type I, there are four possibilities, three in which both links have length 1 and one with one link of length 1 and the other of length 2 . Of course, it follows that this latter subcase can only occur when $f(1) \leq\left|N_{2}\right|$ and $f(2) \leq\left|N_{3}\right|$. Further, any other network in the same MCS has the same structure and each player sponsors the same number of links to each group. Finally observe that a connected CSS network with two links of length 1 is efficient. In Figure 13 examples of these four networks are given. Every arrow representing the external links is solid, because none of the groups receiving an external link becomes is partitioned into more than one component if that link is removed.

Type II networks: these are networks $g$ in which only one group is entirely internally connected, i.e. $\left|\mathcal{N}^{I}(g)\right|=1$. According to property $\mathbf{C}$, 


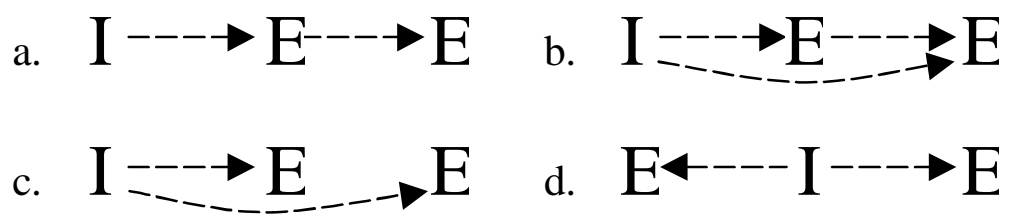

FiguRE 14. Type II networks

again this unique entirely internally connected group is a CSS. A player in an entirely externally connected group can not sponsor a link to the entirely internally connected group, since then he would strictly prefer to replace this link by an internal link, see also Lemma 4. So, any link between the internally connected group and an externally connected group is sponsored by the player in the internally connected group. Now, we consider two subtypes, namely either group 2 in the middle is the unique entirely internally connected group, or group 1 or 3 is. In case it is group 2, then it follows that there can not be a link between a player in group 1 and a player in group 3, because then the player sponsoring the link would strictly prefer to replace this link to a player in his own group. So, all external links are of length 1 and are sponsored by players in group 2. Hence in this case there are links from group 2 to any single player in the groups 1 and 3. Observe that any player in group 2 can sponsor links to one or more players in the other two groups.

In case group 1 or 3 is entirely internally connected, w.l.o.g. we may assume that it is group 1. Now, there are three possibilities, namely (i) all links are sponsored by players in $N_{1}$, (ii) all links to the players in group 2 are sponsored by players in $N_{1}$ and all links to the players in group 3 are sponsored by players in $N_{2}$ and (iii) intermediate cases in which all links to the players in group 2 are sponsored by players in $N_{1}$, while both players in $N_{1}$ and in $N_{2}$ sponsor links to group 3. Clearly, the last two cases can not occur when $f(2)>1$.

Observe that in all the four possibilities of a network of type II, see Figure 14 , there is one central player, as defined in the introduction, i.e. a player $i$ with the property that if we move along a path with subsequently the players $i_{1}, i_{2}, \ldots, i_{k}$ with $i_{1}=i$, then the link from $i_{j}$ to $i_{j+1}$ is sponsored by $i_{j}, j=1, \ldots, k-1$. So, as also stated in the introduction, any network of this type is a generalized CSS: from the central player there is a directed path to any other player in the network. Each path contains at most one player of each group, not counting the central player himself. Clearly, such a network is the single element of an MCS. Of course, a CSS can only arise if $f(1) \leq 1$. Clearly, when $f(1)>f(0)$ (and/or $f(2)>f(0)$ in case $f(2) \leq 1$ ), then a CSS is not efficient. Since the recipient group of each external link becomes partitioned into more than one component if that link is removed, all arrows are dashed. 
Type III networks: These networks $g$ are a hybrid of type 1 and type 2 networks with $1<\left|\mathcal{N}^{I}(g)\right|<m$, i.e. with $m=3$ we have that $\left|\mathcal{N}^{I}(g)\right|=2$ for any type III network. According to property $\mathbf{C}$, again the two entirely internally connected groups are CSSs. Clearly players in the entirely externally connected group can not sponsor links to the entirely internally connected group containing players on these paths, because then these players strictly prefer to replace such external links by internal links. Again, we consider two subtypes, namely either group 2 in the middle is the unique entirely externally connected group, or group 1 or 3 is. In case it is group 2, then all players on any path between two players in group 2 either belong to group 1 or to group 3. Suppose not, then it follows from property $\mathbf{E}$ than there is a player in group 2, say $i$, that observes all others in group 2 by a link sponsored by some player in group 1, and a player in group 2 that observes all others in group 2 by a link sponsored by some player in group 3. However, this implies that there is a link between group 1 and 3 . W.l.o.g. we suppose that this is a player in group 3, say player $k$. However, then player $k$ would strictly prefer to replace his link to group 1 by a link to player $i$ in group 2, contradicting that the network is Nash. W.l.og. suppose that all players on any path between two players in group 2 are in group 1, so there is a link to any player in group 2 sponsored by some player in group 1. Since $N_{1} \in \mathcal{N}^{I}(g)$, it follows that by these links all players in group 1 and 2 are connected to each other. Since also group 3 is entirely internally connected, there will be precisely one link between the players in $N_{1} \cup N_{2}$ and the players in $N_{3}$. For this link there are three possibilities, namely (i) the link is sponsored by player of group 1 (and thus of length 2), (ii), the link is sponsored by a player in group 2 and (iii) the link is sponsored by a player in group 3 to a player in group 2.

Finally, if group 2 is not entirely externally connected, w.l.o.g. we may assume that group 3 is the entirely externally connected group. Now, there are four possibilities, namely (i) all links to the players in group 3 are sponsored by players in group 2 and there is one link from group 1 to group 2 , (ii) all links to the players in group 3 are sponsored by players in group 2 and there is one link from group 2 to group 1, (iii) all links to the players in group 3 are sponsored by players in group 1 and there is one link from group 1 to group 2, and (iv) some links to the players in group 3 are sponsored by players in group 1 and some by players in group 1, while there is a link from group 1 to group 2. Observe that in the last two cases the link between group 1 and group 2 can not go from group 2 to group 1 , because then this player in group 2 is indifferent to this link to group 1 and a link to a player in group 3 receiving a link from a player in group 1, which contradicts property D.

In all these seven possibilities of type III networks, depicted in Figure 15 , both entirely internally connected groups contain a player, say $j_{1}$ and $j_{2}$ that sponsors all links in his own group. The two entirely internally connected groups are CSSs connected to each other, and from $j_{1}$ or $j_{2}$ there is 


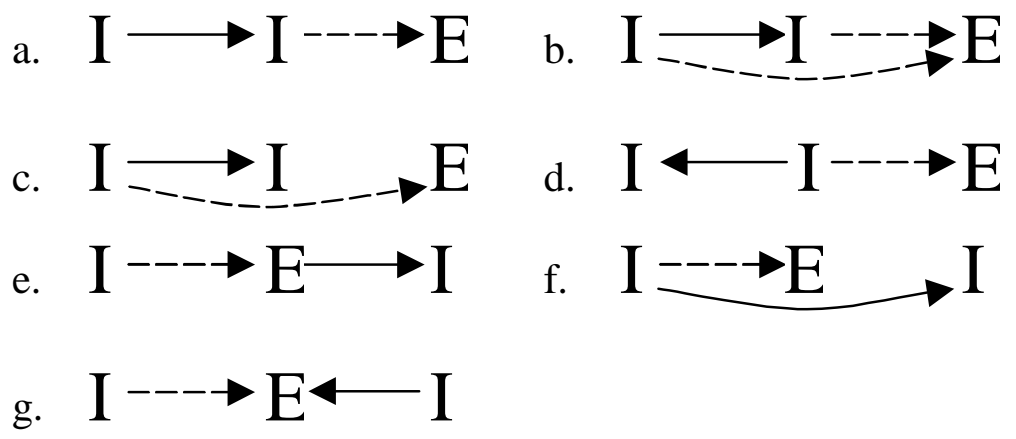

FiguRE 15. Type III networks

a directed path to $i$ for any player $i$ in the externally connected group. We call such a network a hybrid CSS network. So, any network of type III is a hybrid CSS network. Again we have that any other network in the same MCS has the same structure and each player sponsors the same number of links to each group.

The typology given above for $m=3$ also holds when the number of groups is bigger than three. Type I networks are connected CSSs in which any group is organized as a central sponsored star, i.e. $\left|\mathcal{N}^{I}(g)\right|=m$. Such a network is efficient if all external links are of length 1 . However, for low costs of longer links, also external links of length two or more may appear. For instance, when $f(k-1)<\left|N_{k}\right|$ for $k=2, \ldots, m$, then there is an MCS containing type I networks with a link from group 1 to any other group $k, k=2, \ldots, m$. Observe that in such a network all external links are sponsored by a player from group 1.

For low enough values of the external links also networks of type II may appear, for example for $m=4$ see Figure 7 with $f(1)<1$. In such a generalized CSS we have that $\left|\mathcal{N}^{I}(g)\right|=1$ and there is one central player in the unique entirely internally connected group. From this central player there is a (unique) directed path to any other player. A generalized CSS is the unique element of an MCS. A generalized CSS is never efficient in the MG-model.

In case that $1<\left|\mathcal{N}^{I}(g)\right|<m$ we have a hybrid network of type III, for example in case $m=6$ see Figure 11 with $(2,15)$ replaced by $(11,15)$ and $f(4) \leq 1$, which contains four entirely internally connected groups. For $m=6$, two examples with $\left|\mathcal{N}^{I}(g)\right|=m-1$ are given in Figure 16 and Figure 17. Figure 16 illustrates that a network that is a Nash equilibrium can be connected even if $f(1)>1$. Figure 17 shows that links in opposite directions can 'pass' each other: here for instance $(13,1)$ and the links $(11,13)$ and $(8,11)$. However, if $(8,5)$ were to be replaced by $(5,8)$, then this network would not have been in an MCS, because then 5 would have been indifferent between a link to group 1 and to group 3 . If indeed $(8,5)$ is replaced by 


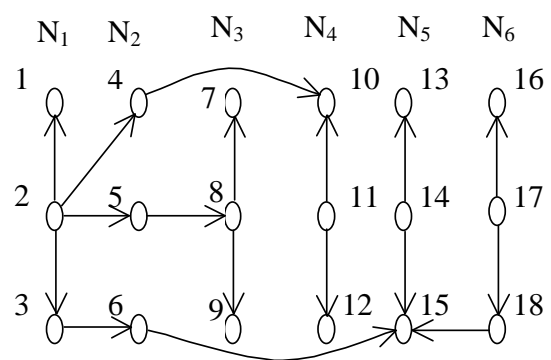

FiguRE 16. Network in an STCS for $1<f(1)<f(2) \leq$ $3 \leq f(3) \leq 6$.

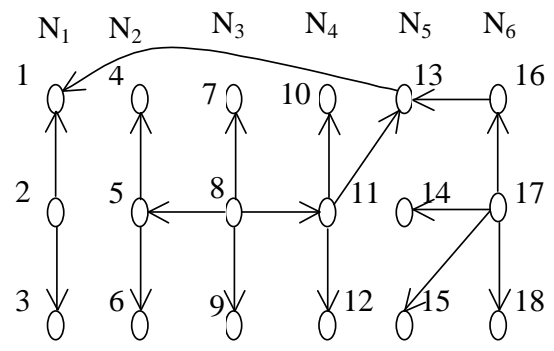

Figure 17. Network in an STCS for $f(4) \leq 3$.

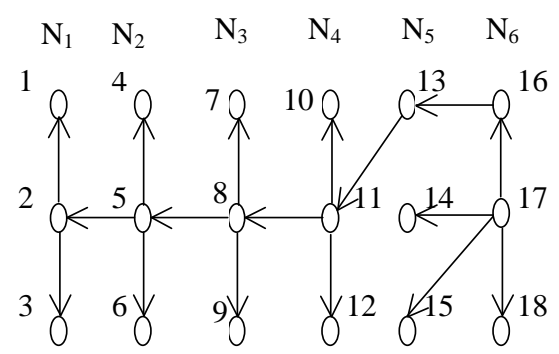

Figure 18. Example of network in STCS for $f(1) \leq 1$.

$(5,8)$, then it is easy to construct a path of updates leading to the network in Figure 18. Figure 18 is, by the way, part of an MCS if $f(1) \leq 1$.

\section{Conclusions}

In this paper we provided a full characterization of the MG model introduced in GGK (they call it the IO model). This model is a one-sided costs, symmetric flow of information model of network formation with a heterogeneous cost functions. We continued the analysis in GGK for a more general 
version of the MG model for one illustrative range of cost functions. We found that if players act as if they are myopically rational, in the long-run it is likely that the system will cycle among a relatively small set of similar networks: a recurrent class. We proved that the static solution concept of minimal curb sets corresponds to the dynamic solution concept of the recurrent class. This result was obtained by proving that each recurrent class contains a super tight curb set (STCS). For these results we focused on an illustrative range of increasing positive cost functions. However, the analysis can be extended to all cost functions, and theorem 5 will continue to hold. The proof for this is not included in the paper, because it is cumbersome and large, while it adds little additional insight.

We found that within a STCS the architecture of the networks is qualitatively identical, although the architectures of different STCSs can be quite different. Nonetheless, also the total variation of architectures over all STCSs is relatively small, because networks all satisfy quite some strict conditions on their architecture if they are part of an STCS. What all STCSs have in common, is that the best reply dynamics can go from any one network in that set to any other network in that set within one period. The set of best responses of players are invariant to the chosen strategies of other players within that set. Moreover, the typical results of centrality and center-sponsorship that is often found in network formation games with homogeneous cost and value functions holds to some extent within these networks. There are generally as many 'centers' as there are groups that are entirely internally connected. For instance: in a situation with 100 groups where half of the groups are entirely internally connected, there are only 50 centers, while the number of players can be arbitrarily large. On a local level we often see the generalized center-sponsored star of GGK (centersponsorship) which is a generalization for cost-heterogeneous models of the center-sponsored star of Bala and Goyal (2000). In fact, all networks that survive in the long run are combinations of generalized center-sponsored stars and connected center-sponsored stars. However not every Nash network that is a combination of generalized center-sponsored stars can survive in the long run, because not all such networks satisfy properties $\mathbf{D}, \mathbf{F}$ and G. In terms of social welfare, the typical STCS network is not efficient, although generally networks from non-singleton STCSs are more efficient that strict Nash networks. The more centers a network has, the higher its level of efficiency.

We further observed that focussing the analysis to strict Nash networks is too restrictive from a dynamic perspective, while studying all Nash networks doesn't exclude some unlikely networks. For models with only few groups, the figures in this paper show some of the richness of architectures that can survive in the long run. The examples give some insight in which architectures may and may not survive under what circumstances.

In terms of our analysis, we were greatly helped by the result of GGK in the two-group MG model, namely that all MCSs are in fact STCSs. By 
proving that this result also holds for the general case the characterization of all MCSs in the $m$-group MG model became much more feasible.

\section{REFERENCES}

[1] V. Bala and S. Goyal (2000), A Strategic Analysis of Network Reliability, Review of Economic Design, 5, 205-228.

[2] V. Bala and S. Goyal (2000), A Non-cooperative Model of Network Formation, Econometrica, 68, 1181-1229.

[3] A-L. Barabási (2002), Linked: The New Science of Networks, Perseus Press, Cambridge MA.

[4] K. Basu and J. Weibull (1991), Strategy Subsets Closed under Rational Behavior, Economics Letters, 36, 141-146.

[5] R. Burt (1992), Structural Holes: The social structure of competition, Harvard University Press, Cambridge, MA.

[6] J. Coleman (1966), Medical Innovation: A diffusion study, Bobbs-Merrill, New York.

[7] B. Dutta and M. Jackson (2000), The stability and efficiency of directed communication networks, Review of Economic Design, 5, 251-272.

[8] A. Falk and M. Kosfeld (2003), It's all about connections: Evidence on network formation, mimeo, University of Zurich.

[9] A. Galeotti, S. Goyal and J. Kamphorst (2003), Network Formation with Heterogeneous Players, Economics Discussion Papers, University of Essex.

[10] S. Goyal (1993), Sustainable Communication Networks, Tinbergen Institute Discussion Paper, 93-250.

[11] M. Granovetter (1974), Getting a job: A study of contacts and careers. University of Chicago Press, Chicago.

[12] H. Haller and S. Sarangi (2001), Nash networks with heterogeneous players, mimeo, VPI.

[13] M. Jackson and A. Wolinsky (1996), A Strategic Model of Social and Economic Networks, Journal of Economic Theory, 71 , 44-74.

[14] M. Jackson (2003), A Survey of Models of Network Formation: Stability and Efficiency, Mimeo, California Institute of Technology, Pasadena.

[15] C. Johnson and R.P. Gilles (2000), Spatial Social Networks, Review of Economic Design, 5, 3, 273-301.

[16] M. McBride (2002), Position-specific Information in Social Networks, University of California.

[17] J. Montgomery (1991), Social networks and labor market outcomes: toward an economic analysis, American Economic Review, 81, 5, 1408-1418.

[18] A. Watts (2001), A Dynamic Model of Network Formation, Games and Economic Behavior, 34, 331-341.

[19] A. Watts (2002), Non-Myopic Formation of Circle Networks, Economics Letters, 74, $272-282$.

[20] D. Watts (2003), Six Degrees: The Science of a Connected Age, W.W. Norton \& Company, New York.

\section{Appendix A. Remaining proofs of lemmas of Section 4}

Proof Lemma 3. First we prove that every group is connected, thus $\gamma_{\ell}(g)=1$ for each $N_{\ell} \in \mathcal{N}$ (see Section 2.3 for notation). Suppose not: then there exists a network $g \in X_{m-1}^{\min }$ and $\ell \in\{1, \ldots, m\}$ such that $\gamma_{\ell}(g)>$ 1. Let some player $i \in N_{\ell}$ update his strategy. By minimality of $g$ and 
$f(0) \leq 1$, player $i$ has a best reply in which he adds at least one internal link towards a player that is within another component. When only this player updates no cycles will be created. Thus there exists a network $g^{\prime} \in X^{\text {min }}$ such that $\delta_{0}\left(g^{\prime}\right)>\delta_{0}(g)$, which contradicts that $g \in X_{0}^{\min }$.

Second we prove that every group is either entirely internally or entirely externally connected, thus for any $\ell$ the number $\sigma_{\ell}(g)$ of internal links within group $N_{\ell}$ is either zero or $\left|N_{\ell}\right|-1$. Suppose not, then there exists some $\ell$ with $\sigma_{\ell}(g) \in\left\{1, \ldots,\left(\left|N_{\ell}\right|-2\right)\right\}$. Thus there exist $i, i^{\prime}, i^{\prime \prime} \in N_{\ell}$, such that $\left\{i, i^{\prime}\right\} \in \bar{g}$ (recall from Section 2.3 that $\bar{g}$ is the collection of (undirected) pairs of players connected by a link) and a path of at least two links between $i$ and $i^{\prime \prime}$ such that on the path all players between $i$ and $i^{\prime \prime}$ do not belong to $N_{\ell}$, i.e. there is a path of undirected pairs $\left\{i, j_{1}\right\}, \ldots,\left\{j_{h}, i^{\prime \prime}\right\}$ in $\bar{g}$ with $h \geq 1$ and $j_{k} \notin N_{\ell}$ for all $k=1, \ldots, h$. If $\left(i, j_{1}\right) \in g$ then player $i$ strictly prefers to replace the link to $j_{1}$ by a link to $i^{\prime \prime}$. Let $b_{i}(g)$ be a best response of $i$ to $g$ in which he indeed replaces this link. Since $g \in X^{\mathrm{min}}$, it follows that $g^{\prime}=g_{-i} \cup b_{i}(g) \in X^{\mathrm{min}}$. Further, it follows that $\delta_{0}\left(g^{\prime}\right)>\delta_{0}(g)$, which contradicts that $X \in X_{m-1}^{\min }$. Analogously we get an contradiction when $\left(i^{\prime \prime}, j_{h}\right) \in g$.

It remains to consider the case that both $\left(j_{1}, i\right)$ and $\left(j_{h}, i^{\prime \prime}\right)$ are in $g$. Here we need to distinguish two cases. First, suppose that $j_{1}$ has a best reply $b_{j_{1}}(g)$ to $g$ containing $\left(j_{1}, i\right)$. Clearly, $j_{1}$ is indifferent between a link to $i$ or to $i^{\prime}$ and thus there also exists a best reply $b_{j_{1}}^{\prime}(g)$ such that $\left(j_{1}, i^{\prime}\right) \in b_{j_{1}}^{\prime}(g)$, Because of this indifference, w.l.o.g. we may assume that $\left(i^{\prime}, i\right) \in g$. However, $i^{\prime}$ is indifferent between a link to $i$ and a link to $i^{\prime \prime}$. Now, suppose that $j_{1}$ switches his link from $i$ to $i^{\prime}$, while simultaneously $i^{\prime}$ switches his link from $i$ to $i^{\prime \prime}$. The resulting network, say $g^{1}$, is not minimal, satisfies $\mathcal{N}^{I}(g) \subseteq \mathcal{N}^{I}\left(g^{1}\right)$ and $\gamma_{\ell}\left(g^{1}\right)>1$ since in $g^{1}$ player $i$ belongs to another component than $i^{\prime}$ and $i^{\prime \prime}$. Suppose that subsequently player $i$ updates. Then $i$ strictly prefers to create an internal link to some player in the component containing $i^{\prime}$ and $i^{\prime \prime}$. Letting player $i$ update, in the resulting network. say $g^{2}$, we have that $\delta_{0}\left(g^{2}\right)>\delta_{0}(g)$. Further, in any best reply of player $j_{1}$, he prefers to delete an external link that he sponsors in the cycle involving $i^{\prime}$ and $i^{\prime \prime}$. Let him do so. Then the resulting network, say $g^{3} \in X^{\min }$ and $\delta_{0}\left(g^{3}\right)=\delta_{0}\left(g^{2}\right)>\delta_{0}(g)$, which contradicts that $g \in X_{m-1}^{\min }$. Finally, we consider the case that $j_{1}$ does not have a best reply to $g$ containing $\left(j_{1}, i\right)$. Then, for any best reply $b_{j_{1}}(g)$, consider the network $g^{\prime}=g_{-j} \cup b_{j_{1}}(g)$. By minimality of $g$ and the updating of one single player, also $g^{\prime}$ is minimal and $j_{1}$ will sponsor at least as many internal links in $g^{\prime}$ as he did in $g$. Then there are two possibilities. First, in $g^{\prime}$ player $j_{1}$ belongs to a different component than $i$. Then $\gamma_{\ell}\left(g^{\prime}\right)>1$, and thus there exists a network $g^{*} \in X^{\text {min }}$ such that $\delta_{0}\left(g^{*}\right)>\delta_{0}\left(g^{\prime}\right)=\delta_{0}(g)$, which again contradicts that $g \in X_{m-1}^{\min }$. Second, $j_{1}$ is connected to $i$ through some player $j^{\prime} \notin N_{\ell}$ with $\left\{i, j^{\prime}\right\} \in \bar{g}^{\prime}$. By arguments already used above it then must hold that $j^{\prime}$ sponsors the link to $i$. Moreover notice that $N^{r}\left(i ; g^{\prime}\right)=\left\{h \in N \mid(h, i) \in g^{\prime}\right\}=N^{r}(i ; g) \backslash\left\{j_{1}\right\}$. Now, repeat all arguments above with $j^{\prime}$ instead of $j_{1}$. Then the only case that did not lead 
to a contradiction until now is that there exists a network $g^{\prime \prime}=g_{-j^{\prime}}^{\prime} \cup b_{j^{\prime}}\left(g^{\prime}\right)$ with $\left(j^{\prime}, i\right) \notin b_{j^{\prime}}\left(g^{\prime}\right)$ and such that $j^{\prime}$ is connected to $i$ through some player $j^{\prime \prime}$ in some other group than $j^{\prime}$ itself, from which we can start all arguments again. However, since $N^{r}\left(i ; g^{\prime \prime}\right)=N^{r}\left(i ; g^{\prime}\right) \backslash\left\{j^{\prime}\right\}=N^{r}(i ; g) \backslash\left\{j_{1}, j^{\prime}\right\}$ and $N^{r}(i ; g)$ is of finite size, the potential number of iterations is finite, which leads again to a contradiction.

Proof Lemma 4. Suppose the lemma is not true. Then $(j, i) \in g$ and $j$ strictly prefers to replace this external link by an internal link, which contradicts that $g \in X_{m-1}^{\min }$.

Proof Lemma 5. Suppose not. Then any group is entirely externally connected and thus there are no internal links within the network. Now, let $C$ be some component of network $g$. Since any group is connected, $C$ consists of a number of groups, say $C=\cup_{\ell \in S} N_{\ell}$ with $S \subset\{1, \ldots, m\}$. Since any group is entirely externally connected, for each group $\ell \in S$ and each player $i \in C \cap N_{\ell}$, there must exist a player $j \in C \backslash N_{\ell}$ such that $\{j, i\} \in \bar{g}$. If $(i, j) \in g$ then $i$ prefers to switch this link to another player $i^{\prime} \in N_{\ell}$, which creates an internal link and contradicts that $g \in X_{m-1}^{\min }$. Hence $(j, i) \in g$. That means that the number of links within $C$ is at least equal to $|C|$, which contradicts the minimality of $g$.

Proof Lemma 6. Suppose not. Then there exists a best reply $b_{i}(g)$ such that $\gamma_{\ell}\left(g^{\prime}\right)>1$ in $g^{\prime}=g_{-i} \cup b_{i}(g)$. Notice that $i$ will not delete any internal link. Letting $j \in N_{\ell}$ update next, we get a minimal network $g^{\prime \prime}=g_{-j}^{\prime} \cup b_{j}\left(g^{\prime}\right)$ for some best reply $b_{j}\left(g^{\prime}\right)$ of $j$ to $g^{\prime}$. Clearly, $\delta_{0}\left(g^{\prime \prime}\right)>\delta_{0}\left(g^{\prime}\right)=\delta_{0}(g)$, because $j$ will create a link towards a player $j^{\prime \prime} \in N_{\ell} \backslash\{j\}$ who in $g^{\prime}$ belongs to another component. This contradicts that $g \in X_{m-1}^{\min }$.

Proof Lemma 7. Again we show the lemma by contradiction. Suppose that $g \in X_{m-1}^{\min }$ is not connected. Since we know already from Lemma 3 that each group is connected and thus each component consists of a number of groups, there exists a component, say $C$, such that $|C| \geq \max _{N_{\ell} \in \mathcal{N}}\left|N_{\ell}\right|$. Since $g$ is not connected there also exists numbers $\ell$ and $k \in\{\ell-1, \ell+1\}$ such that $N_{\ell} \subseteq N \backslash C$ and $N_{k} \subseteq C$. Then each player $i \in N_{\ell}$ has at least one best reply to $g$ in which he sponsors a link to a player in group $N_{k}$, because by assumption $f(1) \leq \max _{N_{\ell} \in \mathcal{N}}\left|N_{\ell}\right| \leq|C|$. Let one of these players update by playing this best reply and let $g^{\prime}$ be the resulting network. Clearly, $i$ will not delete any internal link when updating his strategy. So, if $i$ does not delete a link of length one in his best reply, then we have that $\delta_{1}\left(g^{\prime}\right)>\delta_{1}(g)$ and $\delta_{0}\left(g^{\prime}\right)=\delta_{0}(g)$, contradicting that $g \in X_{m-1}^{\min }$. If $i$ deletes a link of length one in his best reply to $g$, then this deleted link must be to the other neighboring group in $\{\ell-1, \ell+1\}$ not equal to $k$. Denote the resulting network $g^{1}$ and notice that $g^{1} \in X$ and satisfies $\delta_{1}\left(g^{1}\right)=\delta_{1}(g)$ and $\delta_{0}\left(g^{1}\right)=\delta_{0}(g)$. Moreover, $g^{1}$ is not connected. On the other hand 
group $N_{k}$ is now connected with $N_{\ell}$. Now repeat the procedure with $C$ the component of $g^{1}$ containing there two groups. This leads either to a contradiction as above when the updating player does not delete a link of length 1 , or to a next network $g^{2}$ with $\delta_{1}\left(g^{2}\right)=\delta_{1}\left(g^{1}\right)$ and $\delta_{0}\left(g^{2}\right)=\delta_{0}\left(g^{1}\right)$ and in which the next group is connected to the component. Since there are only a finite number of groups to any side of group $\ell$, within a finite number of steps this iterative procedure will reach a network in which the updating player will not delete a link of length 1 , which leads to a contradiction as above. Hence $g$ must be connected.

Proof Lemma 8. Suppose not. Let any one sponsor of such link update, and call the resulting graph $g^{\prime}$. Then there are two cases. Either $g^{\prime}$ is disconnected, in which case by earlier arguments a link of length 1 can be created such that the resulting network $g^{\prime \prime}$ has $\delta_{1}\left(g^{\prime \prime}\right)>\delta_{1}(g)$ and $\delta_{0}\left(g^{\prime \prime}\right)=\delta_{0}(g)$. Or $g^{\prime}$ is connected, but then the only change is that a longer link is replaced by a shorter link. Both possibilities contradict that $g \in X_{m-1}^{\min }$.

Proof Lemma 10. The proof follows from an iterative procedure very similar to that of Lemma 7. Consider any network $g \in X_{m-1}^{\min } \backslash \widehat{X}_{m-1}^{\min }$, thus $g$ contains at least one unstable link of length 1 and let $K$ be the number of these unstable links. So, there exists a link $(i, j)$ and numbers $\ell$ and $k \in\{\ell-1, \ell+1\}$ such that $i \in N_{\ell}$ and $j \in N_{k}$, such that $(i, j) \notin b_{i}(g)$ for any best reply $b_{i}(g) \in B_{i}(g)$. Now, suppose player $i$ sponsors two or more links to group $k$. Then $N_{k}$ must be entirely externally connected, otherwise there is a cycle which contradicts the minimality of $g$. Since any group belongs to the same component, it follows that the players in $N_{k}$ to which $i$ sponsors links are connected via those links. Then it follows from Lemma 6 that all these links must be stable. So, if $(i, j)$ is unstable, then $j$ is the unique player in $N_{k}$ to which $i$ sponsors a link. Hence, player $i \in N_{\ell}$ can sponsor at most two unstable links, one to group $\ell-1$ and one to $\ell+1$. First consider the case that $i$ sponsors only one unstable link. W.l.o.g. we assume that $\ell>1$ and $k=\ell-1$. Let player $i$ update and call the resulting graph $g^{\prime}$. Since $g$ is connected and $(i, j)$ is unstable, we must have that $f(1)$ is larger than the component of $g^{\prime}$ not containing $i$, and thus by the assumption that $f(1) \leq \max _{N_{\ell} \in \mathcal{N}}\left|N_{\ell}\right|$, it must hold that $f(1)$ is at most equal to the component in $g^{\prime}$ containing player $i$. Hence, each player in group $\ell-1$ has an incentive to create a link to $i$. Let $i_{1} \in N_{\ell-1}$ do so and let $g^{1}$ be the resulting network. Then either $g^{1} \in X_{m-1}^{\min }$ and has one unstable link less than $g$, or $i_{1}$ has deleted an unstable link to a player in group $N_{\ell-2}$. In the first case we have that the number of unstable links in $g^{1}$ is $K-1$. Otherwise, any player in $N_{\ell-2}$ has an incentive to create a link to $i_{1}$. Let some player $i_{2} \in N_{\ell-2}$ do so and let $g^{2}$ be the resulting network. Then again, either $g^{2} \in X_{m-1}^{\min }$ and has one unstable link less than $g$, or $i_{2}$ has deleted an unstable link to a player in group $N_{\ell-3}$. Since $\ell$ is finite, continuing this iterative procedure 
when latter case occurs, we obtain within a finite number of steps a network in $X_{m-1}^{\min }$ and having at most $K-1$ unstable links.

Finally we consider the case that $i$ sponsors two unstable links, i.e. one to group $\ell-1$ and one to group $\ell+1$, say to player $j_{1} \in N_{\ell-1}$ and to $j_{2} \in N_{\ell+1}$. Let player $i$ update and call the resulting graph $g^{\prime}$. Since $g$ is connected and both $\left(i, j_{1}\right)$ and $\left(i, j_{2}\right)$ are unstable, we must have that $f(1)$ is larger than the component of $g^{\prime}$ not containing $i$, and thus by the assumption that $f(1) \leq \max _{N_{\ell} \in \mathcal{N}}\left|N_{\ell}\right|$, it must hold that $f(1)$ is at most equal to the component in $g^{\prime}$ containing player $i$. Hence, each player in group $\ell-1$ has an incentive to create a link to $i$ and each player in group $\ell+1$ has an incentive to create a link to player $i$. Moreover, since $f(x)>f(1)$ for all $x \geq 2$, no player in any group $\ell-y(\ell+y)$ will sponsor a link to any player in group $\ell+1$ (respectively $\ell-1$, for all $y>0$. Therefore we can apply the procedure for the case of one unstable link to both sides of group $N_{\ell}$ in case of two unstable links, which will lead in a finite number of steps to a network in $X_{m-1}^{\min }$ with $K-2$ unstable links.

Consequently, for any network in $X_{m-1}^{\min } \backslash \widehat{X}_{m-1}^{\min }$ the procedure leads to a network in $X_{m-1}^{\min }$ with a smaller number of unstable links. Since the number of unstable links is finite, starting with some network in $X_{m-1}^{\min } \backslash \widehat{X}_{m-1}^{\min }$, iteration of the procedure will lead within a finite number of iterations to a network without any unstable link of length one.

Proof Lemma 11. Since any network $g \in \widehat{X}_{m-1}^{\min }$ is a Nash network, it follows from Lemma 1 that any player $i$ sponsors precisely one link to any component of the network $g_{-i}$ in all his best replies to $g$. Moreover, when $k$ is the number of components, the link to a component $C_{h}, h=1, \ldots, k$, of $g_{i}$ is to one of the players in $D_{h}$, the set of players in $C_{h}$ that are closest to $i$. So, when in $g$, player $i$ sponsors an internal link to a player in some component $C_{h}$ of $g_{-i}$, he will sponsor precisely one internal link to $C_{h}$ in any best reply to $g$. Hence in any best reply player $i$ sponsors the same number of internal links.

We now consider the number of links that some player $i$ in some group $N_{\ell}$ sponsors a link $(i, j)$ to some group $N_{\ell^{\prime}}, \ell^{\prime} \neq \ell$. W.l.o.g. we assume that $h<\ell$. Suppose that the lemma does not hold. Since the network $g$ is Nash, it follows again from Lemma 1 that for any component $C_{h}$ player $i$ sponsors precisely one link to the subset of players $D_{h}$ of $C_{h}$ in any best reply of $i$ to $g_{-i}$. Let $b_{i}(g)$ be a best reply of $i$. Since any player in $D_{h}$ is on equal distance of $i$, it must hold that when $(i, j)$ is not in $b_{i}(g)$, then either $(i, j)$ is replaced by either a link to another player in group $\ell^{\prime}$, or by a link to a player in group $\ell^{\prime \prime}=2 \ell-\ell^{\prime}>\ell$. In the first case the number of links sponsored by $i$ to group $\ell$ is equal as in $g$.

It remains to consider the second case. Let $j^{\prime}$ be the player in $N_{\ell^{\prime \prime}}$ to which $i$ sponsors a link in $b_{i}(g)$ instead of his link $(i, j)$ in $g$. Since $j$ and $j^{\prime}$ are both in the same component $C_{h}$ of $g_{-i}$, there is a path between $j$ 
and $j^{\prime}$ in $g_{-i}$. Moreover, this path does not contain players closer to $i$. So, this path contains a link $\left(j_{1}, j_{2}\right)$ with $j_{1} \in N_{r}$ for some $r \leq \ell^{\prime}$ and $j_{2} \in N_{s}$ for some $s \geq \ell^{\prime \prime}$. Now, suppose $i$ updates his strategy and replaces his link $(i, j)$ by $\left(i, j^{\prime}\right)$. Next, let $j_{1}$ update his strategy. Clearly, this player now strictly prefers to replace his link to $j_{2}$ by the shorter link to $i$. However, this contradicts that $g \in X_{m-1}^{\min }$.

Proof Lemma 12. Suppose the lemma is not true. Then there must exist three distinct players $j, j^{\prime}, j^{\prime \prime} \in N_{\ell}, N_{\ell} \in \mathcal{N}^{E}(g)$, and two different players $i$ and $i^{\prime}$ not in $N_{\ell}$, such that $j$ observes $j^{\prime}$ through the link $(i, j)$ and $j^{\prime \prime}$ through $\left(i^{\prime}, j\right)$. Since $g \in \widehat{X}_{m-1}^{\min }$, player $i$ has a best reply to $g$ containing $(i, j)$. Since $j$ and $j^{\prime \prime}$ are in the same component of $g_{-i}$ and have equal distance to $i$, it follows from Lemma 1 that there is also a best reply $b_{i}(g)$ of $i$ containing $\left(i, j^{\prime \prime}\right)$, thus $\left(i, j^{\prime \prime}\right) \in b_{i}^{\prime}(g)^{16}$. Likewise player $i^{\prime}$ has a best reply $b_{i^{\prime}}(g)$ such that $\left(i^{\prime}, j^{\prime}\right) \in b_{i^{\prime}}(g)$. Now consider the network $g^{\prime}=\left(g \backslash\left(g_{i} \cup g_{i^{\prime}}\right)\right) \cup b_{i}(g) \cup b_{i^{\prime}}(g)$. Then $g^{\prime}$ is not minimal, since $i$ and $i^{\prime}$ are connected via exactly two paths and $j$ is isolated. Now let $j^{\prime}$ update. Then $j^{\prime}$ creates one internal link to $j$ in the resulting network $g^{\prime \prime}$. Consequently, player $i$ has a best reply $b_{i}\left(g^{\prime \prime}\right)$ to $g^{\prime \prime}$ such that $b_{i}\left(g^{\prime \prime}\right)=g_{i}^{\prime \prime} \backslash\left\{\left(i, j^{\prime \prime}\right)\right\}$. When $i$ updates according to this best reply, the resulting graph is minimal, connected and contains more internal links than $g$, which contradicts that $g \in X_{m-1}^{\min }$.

Proof Lemma 13. Consider $g \in \widehat{X}_{m-1}^{\min }$. Let $g_{-N_{\ell}}$ be the network $g$ minus all the internal links of group $\ell$. Notice that since $g$ is a Nash network, for all $g^{\prime} \in \mathcal{G}$ such that $g_{-N_{\ell}}^{\prime}=g_{-N_{\ell}}$, no player $i \in N_{\ell}$ will strictly prefer to change his strategy with respect to any player outside group $\ell$. That is: he does not strictly prefer to add, delete or replace a link to any player but players in group $\ell$ itself. Therefore in this proof we may and do assume that, as long as $g_{-N_{\ell}}$ remains unchanged, none of the players in $N_{\ell}$ will change his strategy with respect to any player outside group $\ell$. Similarly, given any minimal connected network $g^{\prime}$ such that $g_{-N_{\ell}}^{\prime}=g_{-N_{\ell}}$, then the sets of best replies of any player $i \notin N_{\ell}$ stays unchanged, because $N_{\ell}$ is internally connected in $g^{\prime}$. Now consider any one group $N_{\ell} \in \mathcal{N}^{I}(g)$ that is not a CSS. Let only players inside group $\ell$ update. Then, by Bala and Goyal (2000) there exist a sequence of best replies that leads to $N_{\ell}$ being a CSS. Name the resulting graph $g^{1}$. Since $g_{-N_{\ell}}$ remained unchanged, and because $N_{\ell} \in \mathcal{N}^{I}\left(g^{1}\right)$, $g^{1} \in \widehat{X}_{m-1}^{\min }$. By iteration there exists $g^{\prime \prime} \in \widetilde{X}_{m-1}^{\min }$, and thus $\widetilde{X}_{m-1}^{\min } \neq \emptyset$.

Proof Lemma 14. Let $g^{*}$ be a network in $\widetilde{X}_{m-1}^{\min }$. Now, suppose the lemma is not true. Then there must be a network $g \in B\left(g^{*}\right)$, such that $B(g) \neq B\left(g^{*}\right)$. So, let $Y \subseteq B\left(g^{*}\right)$ be the nonempty subset of networks

\footnotetext{
${ }^{16}$ Note that $(i, j) \notin b_{i}^{\prime}(g)$, since sponsoring both $(i, j)$ and $\left(i, j^{\prime \prime}\right)$ would create a cycle given $g_{-i}$.
} 
given by:

$$
Y=\left\{g \in B\left(g^{*}\right) \mid B(g) \neq B\left(g^{*}\right)\right\} .
$$

By definition, in any network $g \in Y$ there is at least one player $i$ that plays an other strategy than in $g^{*}$, otherwise $g=g^{*}$ and so $B(g)=B\left(g^{*}\right)$. For $g \in Y$, denote the number of players that play a different strategy in network $g$ than in $g^{*}$ by

$$
\phi(g)=\left|\left\{i \in N \mid g_{i} \neq g_{i}^{*}\right\}\right| .
$$

and define $Y^{*} \subseteq Y$ as the subset of networks in $Y$ such that the number of players that play a strategy different from their strategy in $g^{*}$ is minimal, i.e.

$$
Y^{*}=\left\{g \in Y \mid \phi(g)=\min _{g^{\prime} \in Y} \phi\left(g^{\prime}\right)\right\} .
$$

Clearly $\phi(g) \geq 1$ for any $g \in Y^{*}$. Let $g^{1}$ be some network in $Y^{*}, i$ a player such that $g_{i}^{1} \neq g_{i}^{*}$ and define $g^{0}=g_{-i}^{1} \cup g_{i}^{*}$. We now proceed in two steps. First we show that $g^{1} \in X_{m-1}^{\min }$ and $g^{0} \in \widehat{X}_{m-1}^{\min }$. Next, we show that these properties contradict that $B\left(g^{1}\right) \neq B\left(g^{*}\right)$.

To show the first step, recall that $g^{*} \in \widetilde{X}_{m-1}^{\min }$ and thus $g^{*}$ is Nash. Hence $g_{i}^{*} \in B_{i}\left(g^{*}\right)$. Moreover, $g^{1} \in Y \subset B\left(g^{*}\right)$ and thus $g_{j}^{1} \in B_{j}\left(g^{*}\right)$ for all $j \neq i$. Hence, $g_{j}^{0} \in B_{j}\left(g^{*}\right)$ for all $j$ and thus $g^{0} \in B\left(g^{*}\right)$. Since $g_{i}^{0}=g_{i}^{*}$ and $i$ plays different from $g_{i}^{*}$ in $g^{1}$, by construction it holds that $\phi\left(g^{0}\right)=\phi\left(g^{1}\right)-1$. However, since $g^{1} \in Y^{*}$ this implies that $g^{0} \notin Y$ and thus $B\left(g^{0}\right)=B\left(g^{*}\right)$. So, $g^{0} \in B\left(g^{*}\right)=B\left(g^{0}\right)$ and thus $g^{0}$ is a Nash network. Further, since $g^{*} \in \widetilde{X}_{m-1}^{\min } \subseteq \widehat{X}_{m-1}^{\min } \subset X^{\text {min }}$, by definition of $X^{\text {min }}$ we have that $g^{*}$ is minimal connected. Since both $g^{0}, g^{1} \in B\left(g^{*}\right)$, it follows from Lemma 11, that each player sponsors in both $g^{0}$ and $g^{1}$ the same number of links as in $g^{*}$ to each group. Hence, the total number of links is the same in each of the three networks. Hence $g^{0}$ is minimal connected, since otherwise there is at least once cycle which contradicts that $g^{0}$ is Nash. To show that also $g^{1}$ is minimal connected, suppose not. Then $g^{1}$ contains at least one cycle. Hence, there is at least one player, say player $j$, that sponsor a link within a cycle. For this player $j$, let $g^{\prime}$ be the network given by $g^{\prime}=g_{-j}^{1} \cup g_{j}^{*}$. Then, analogous to the reasoning for $g^{0}$, it follows that $B\left(g^{\prime}\right)=B\left(g^{*}\right)$ and thus $g_{j}^{1} \in B_{j}\left(g^{*}\right)=B_{j}\left(g^{\prime}\right)$. So, $g_{j}^{1}$ is a best reply to $g^{\prime}$, which contradicts that $j$ sponsors a link in a cycle. Hence, also $g^{0}$ and $g^{1}$ are minimal connected and thus belong to $X^{\mathrm{min}}$. Again, it follows from Lemma 11 that $\delta_{k}\left(g^{1}\right)=\delta_{k}\left(g^{0}\right)=\delta_{k}\left(g^{*}\right)$ for all $k$ and thus both $g^{0}, g^{1} \in X_{m-1}^{\min }$. Since $g^{0}$ is Nash, we also have that $g^{0} \in \widehat{X}_{m-1}^{\min }$, which completes the first step.

To show the second step, consider $g^{0}=g_{-i}^{1} \cup g_{i}^{*}$ and let player $i$ update to $g_{i}^{1} \in B_{i}\left(g^{*}\right)$. Clearly, $B_{i}\left(g^{1}\right)=B_{i}\left(g^{0}\right)=B_{i}\left(g^{*}\right)$. Since $B\left(g^{1}\right) \neq B\left(g^{*}\right)=$ $B\left(g^{0}\right)$, there must be a player $j \neq i$ such that $B_{j}\left(g^{1}\right) \neq B_{j}\left(g^{0}\right)$. Now, let $C_{1}\left(g^{0}\right), \ldots, C_{k}\left(g^{0}\right)$ be the components of $g_{j}^{0}$ not containing player $j$ and 
$C_{1}\left(g^{1}\right), \ldots, C_{m}\left(g^{1}\right)$ the components of $g_{j}^{1}$ not containing player $j$. Since both $g^{0}$ and $g^{1}$ are minimally connected and $g_{j}^{0}=g_{j}^{1}$ it follows that $k=m=$ $\left|\left\{\left(j, j^{\prime}\right) \mid\left(j, j^{\prime}\right) \in g_{j}^{0}\right\}\right|$ and that for any $h=1, \ldots, k$ there exists precisely one player $j_{h} \in C_{h}\left(g^{0}\right) \cap C_{h}\left(g^{1}\right)$ such that $\left(j, j_{h}\right) \in g_{j}^{0}=g_{j}^{1}$. Referring to the notation of Lemma 1 we have that $j_{h} \in D_{h}\left(g^{0}\right)$ for any $h=1, \ldots, k$ and that

$$
B_{j}\left(g^{0}\right)=\Pi_{h=1}^{m} D_{h}\left(g^{0}\right)
$$

Now, let $D_{h}\left(g^{1}\right)$ be the sets as defined in Lemma 1 with respect to $g^{1}$. Given the independency of whether or not it is a best reply to sponsor a link to any of the components of $C_{h}\left(g^{1}\right)$, it suffices to consider any component separately. Since $B_{j}\left(g^{1}\right) \neq B_{j}\left(g^{0}\right)$, there is at least one $h$ with $D_{h}\left(g^{0}\right) \neq$ $D_{h}\left(g^{1}\right)$. Then, for such an index $h$, there are three possibilities.

1. $D_{h}\left(g^{1}\right)=\emptyset$.

2. $D_{h}\left(g^{1}\right) \neq \emptyset$ and $j_{h} \notin D_{h}\left(g^{1}\right)$.

3. $D_{h}\left(g^{1}\right) \neq \emptyset$ and $j_{h} \in D_{h}\left(g^{1}\right)$.

We first consider Case 1 . In this case player $j$ does not want to sponsor a link to $C_{h}\left(g^{1}\right)$. Since $g^{0}$ is a Nash network, it then must be that $j$ observes less players via the link $\left(j, j_{h}\right)$ in $g^{1}$ than in $g^{0}$. Since $g^{1}$ is obtained from $g^{0}$ because player $i$ revised his strategy, it must be that player $i$ has removed himself from the set of players that $j$ observes via $j_{h}$ by replacing some link, say $\left(i, i^{\prime}\right)$ by another link $\left(i, i^{\prime \prime}\right)$. This can only be the case when $i^{\prime}$ and $i^{\prime \prime}$ belong to an entirely externally connected group, and the path from $i^{\prime}$ to $i^{\prime \prime}$ contains $\left(j, j_{h}\right)$. Consider $g^{1}$ and let player $j$ update. In the resulting network $g^{2}$ player $i^{\prime}$ does not observe $i^{\prime \prime}$. So, when $i^{\prime}$ gets the opportunity to update, he creates an internal link so that he observes $i^{\prime \prime}$ again. The resulting network is minimal, and has more internal links than $g^{*} \in X_{m-1}^{\min }$ : a contradiction. So, we conclude that $D_{h} \neq \emptyset$ for any component $h$ and therefore it holds that for any best reply of $j$ to $g^{1}$, the resulting network is minimal.

In case 2 it must hold that any player in $D_{h}\left(g^{1}\right)$ is closer to player $j$ than $j_{h}$. Now, when player $j$ plays a best reply to $g^{1}$, he replaces his link to $j_{h}$ by a shorter link. So, for any best the resulting network is minimal and has at least one link shorter than $g^{1}$. This contradicts that $g^{1} \in X_{m-1}^{\min }$.

Case 3 contains 2 subcases, namely

3a. $D_{h}\left(g^{1}\right) \backslash D_{h}\left(g^{0}\right) \neq \emptyset$.

3b. $D_{h}\left(g^{1}\right) \subset D_{h}\left(g^{0}\right)$.

In Case 3a, let $j^{\prime} \in D_{h}\left(g^{1}\right) \backslash D_{h}\left(g^{0}\right)$ be a new alternative for the link of player $j$ to $j_{h}$. Let $\ell$ and $\ell_{h}$ be the groups of the players $j$, respectively $j_{h}$. Without loss of generality we assume that $\ell_{h}<\ell$. Since $j_{h} \in D_{h}\left(g^{1}\right)$ and any player in $D_{h}\left(g^{1}\right)$ has the same distance to $j$, it follows that $j^{\prime} \in N_{\ell^{\prime}}$ with either $\ell^{\prime}=\ell_{h}$ or $\ell^{\prime}-\ell=\ell-\ell_{h}>0$. We first consider the latter case (thus $\ell^{\prime}>\ell>\ell_{h}$ ). Since $j_{h}$ and $j^{\prime}$ are both in $C_{h}\left(g^{1}\right)$, there exists a path in $g^{1}$ between $j_{h}$ and $j^{\prime}$ that does not include the link $\left(j, j_{h}\right)$. This 
path includes a link between two players $k \in N_{\ell_{k}}$ and $k^{\prime} \in N_{\ell_{k^{\prime}}}$ with $\ell_{k} \leq \ell$ and $\ell_{k^{\prime}}>\ell$. Now again there are two subcases. (a) $\ell_{k}=\ell$. In this case $j$ prefers an internal link to $k$ above an external link to $j_{h}$ or $j^{\prime}$, contradicting that $j_{h}, j^{\prime} \in D_{h}\left(g^{1}\right)$. (b) $\ell_{k}<\ell$. Since $\ell_{k}<\ell$ and $\ell_{k^{\prime}}>\ell$, we have that $\ell_{k^{\prime}}-\ell_{k} \geq 2$. Now, either $\left(k, k^{\prime}\right) \in g^{1}$ or $\left(k^{\prime}, k\right) \in g^{1}$. Since in $g^{1}$ player $k$ is connected to $j$ via $j_{h}$ and thus $k$ and $j$ belong to the same component in $g_{-k^{\prime}}^{1}$ the link $\left(k^{\prime}, k\right)$ can not be in $g^{1}$, because the player $k^{\prime}$ would strictly prefer to replace this link $k^{\prime}$ by the shorter link to $j$, contradicting that $g^{1} \in X_{m-1}^{\min }$. To apply the same reasoning to exclude $\left(k, k^{\prime}\right)$, let $g^{\prime}$ be the network obtained by replacing the link from $j$ to $j_{h}$ by a link to $j^{\prime}$. Clearly, in $g^{\prime}$ player $k$ strictly prefers to replace his link to $k^{\prime}$ to the shorter link to $j$.

We now consider Case 3a with $\ell^{\prime}=\ell_{h}$. If $N_{\ell_{h}} \in \mathcal{N}^{I}\left(g^{0}\right)$, then it follows that $N_{\ell_{h}} \subseteq D_{h}\left(g^{0}\right)$, which excludes that $j^{\prime} \in D_{h}\left(g^{1}\right) \backslash D_{h}\left(g^{0}\right)$. The same holds when $N_{\ell_{h}} \in \mathcal{N}^{E}\left(g^{0}\right)$ and $j_{h}$ does not observe his group members via $j$ (see Lemma 12). So, it remains to consider the possibility that $N_{\ell_{h}} \in$ $\mathcal{N}^{E}\left(g^{0}\right)$ and $j_{h}$ observes in $g^{0}$ all other players in $N_{\ell_{h}}$ via $j$. So, in $g^{0}$ player $j$ has no alternatives for his link to $j_{h}$. Consider $g^{1}$ and let the players $i$ and $j$ update simultaneously to respectively his best reply $g_{i}^{0}=g_{i}^{*}$ to $g^{1}$ and player $j$ to a best reply in which he switches his link from $j_{h}$ to $j^{\prime}$. In the resulting network, say $g^{2}$, there is a cycle containing player $j$, while $j_{h}$ and $j^{\prime}$ do not observe each other. Now, let $j_{h}$ update. Then he creates an internal link to observe $j^{\prime}$. Finally when $j$ updates he will delete a link in the cycle of the network. The resulting network, say $g^{3}$, is therefore minimal and it has more internal links than $g^{*}$, contradiction that $g^{*} \in X_{m-1}^{\min }$. This concludes Case 3a.

Finally, we consider Case 3b, where some of the alternatives in $D_{h}\left(g^{0}\right)$ do not belong to $D_{h}\left(g^{1}\right)$. Arguments here are similar to Case 3a. First notice that in $g^{0}$, player $j$ cannot be indifferent between two players in different groups. Hence, in $g^{0}$, player $j$ is indifferent between a link to $j_{h}$ and a link to any other $k \in N_{\ell_{h}}$. Let $k \in N_{\ell_{h}} \backslash D_{h}\left(g^{1}\right)$. In $g^{1}, j$ is not indifferent. By Lemma 6 there is still a path between $j_{h}$ and $k$. Since in $g^{1}$ player $j$ strictly prefers not to replace his link to $j^{\prime}$ by a link to $k$ the link $\left(j, j_{h}\right)$ must be part of the path between $j_{h}$ and $k$ in $g^{1}$. Consider $g^{0}$, and let both $i$ and $j$ update. Let $i$ choose $g_{i}^{1}$ and let $j$ replace $\left(j, j_{h}\right)$ by $(j, k)$. In the resulting network, say $h^{0}$, a cycle is created, and as a result $j_{h}$ and $k$ do not observe each other. Let $k$ update, he will sponsor an additional internal link so that he will observe $j_{h}$ again. Finally let $j$ update: he will remove a link from the cycle. The resulting network, say $h^{1}$, is minimal, and contains more internal links that $g^{*}$ : a contradiction with $g^{*} \in X_{m-1}^{\min }$.

\section{Appendix B. Proof Lemma 16}

In this appendix we will show Lemma 16 by proving two additional lemmas, from which it follows immediately. First, recall from Section 2.3 that 
for $i \in N_{\ell}, N^{R}(i ; g)$ is the set of players that sponsor a link to player $i$, satisfying that any other player $i^{\prime} \in N_{\ell}$ observed by $i$ will also be observed by $i$ when any player $j \in N^{R}(i ; g)$ cancels his link to $i$, i.e. the links of the players in $N^{R}(i ; g)$ are not essential for player $i$ to be whether or not connected to the other players in his group. Notice that this implies that in a minimal network the set $N^{R}(i ; g)$ can not contain any player of the set $N_{\ell}$ itself. Further, let $N^{R}\left(N_{\ell} ; g\right)=\cup_{i \in N_{\ell}} N^{R}(i ; g)$. Then the next lemma states that when $g \in \widetilde{X}_{m-1}^{\min }$ and $N_{\ell}$ is entirely externally connected, this set contains at most one player. We first give an example, showing that the assumption that $g \in \widetilde{X}_{m-1}^{\min }$ is violated when $N^{R}\left(N_{\ell} ; g\right)$ contains multiple players.

Example 10. Consider Figure 8 but with link $(5,1)$ replaced by link $(1,7)$ and suppose that this modified network $g \in X$ for some MCS $X$. Observe that any entirely internally connected set is a CSS, which is needed for the network to be in $\widetilde{X}_{m-1}^{\min }$, and that $N^{R}\left(N_{3} ; g\right)=\{1,5\}$ and thus contains two players. However, in this network player 1, who is further away from group 3 than player 5 , prefers to replace his link to group 3 by a link to player 5 . So, the link $(1,7)$ of length two is unstable and hence by Lemma $8 g$ is not in $X_{m-1}^{\min }$ and thus also not in $\widetilde{X}_{m-1}^{\min }$. This is a contradiction.

Lemma 17. Let $g \in \widetilde{X}_{m-1}^{\min }$ and $N_{\ell} \in \mathcal{N}^{E}$. Then $\left|N^{R}\left(N_{l} ; g\right)\right| \leq 1$.

Proof. Suppose that $\left|N^{R}\left(N_{l} ; g\right)\right|>1$. Then there exist two distinct players $i, i^{\prime} \notin N_{\ell}$ and two players $j, j^{\prime} \in N_{\ell}$, such that $i \in N^{R}(j ; g)$, and $i^{\prime} \in$ $N^{R}\left(j^{\prime} ; g\right)$. Notice that either $j=j^{\prime}$ or $j \neq j^{\prime}$.

We first consider the latter case. Then $j$ and $j^{\prime}$ are connected via a unique path that does neither include $(i, j)$, nor $\left(i^{\prime}, j^{\prime}\right)$. Thus there exist players $i_{1}, i_{2} \notin N_{\ell}, i_{1} \notin N^{R}(j ; g)$ and $i_{2} \notin N^{R}\left(j^{\prime} ; g\right)$ such that $\left(i_{1}, j\right),\left(i_{2}, j^{\prime}\right) \in g$ and $\left\{i, i^{\prime}\right\} \cap\left\{i_{1}, i_{2}\right\}=\emptyset$. Let $i \in N_{k}, i^{\prime} \in N_{k^{\prime}}, i_{1} \in N_{k_{1}}$ and $i_{2} \in N_{k_{2}}$. W.l.o.g we assume that $k_{1}>\ell$. Suppose $k_{2}<\ell$. Since the path connecting $j$ and $j^{\prime}$ contains both $i_{1}$ and $i_{2}$, it then must also contain a link $\left\{j_{1}, j_{2}\right\} \in \bar{g}$ with $j_{1} \in N_{\ell_{1}}$ and $j_{2} \in N_{\ell_{2}}$ for some $\ell_{2}<\ell \leq \ell_{1}$, such that $j_{1}$ observes $j^{\prime}$ via $j_{2}$. Notice that $\ell_{2} \neq \ell$, otherwise Lemma 12 is violated. However, then $g$ is not Nash, because if $\left(j_{1}, j_{2}\right) \in g$ (respectively $\left(j_{2}, j_{1}\right) \in g$, then $j_{1}$ $\left(j_{2}\right)$ would strictly prefer to replace his link to $j_{2}\left(j_{1}\right)$ by a link to $j^{\prime}(j)$. Hence $k_{2}>\ell$ if $k_{1}>\ell$. Next suppose that $k \geq \ell$. If $k=\ell$, then $\sigma_{\ell}(g)>0$, which contradicts that $N_{\ell}$ is entirely externally connected. Further, when $\ell<k \leq k_{1}$ (respectively $\ell<k_{1}<k$, then $g$ is not a Nash network, since $i_{1}(i)$ would then be strictly better of by switching his link from $j$ to $i$ $\left(i_{1}\right)$. Hence, it follows that $k<\ell$ must hold. Analogously, $k^{\prime}<\ell$, since $k_{2}>\ell$. So, it follows both $k$ and $k^{\prime}$ are smaller than $\ell$. However, when $k \leq k^{\prime}<\ell$ (respectively $k^{\prime}<k<\ell$ ), then $i\left(i^{\prime}\right)$ would be better of by switching his link from $j$ to $i^{\prime}(i)$, contradicting that $g$ is a Nash network. Hence, $\left|N^{R}\left(N_{l} ; g\right)\right|>1$ contradicts that $g$ is a Nash network. 
Similarly the case $j=j^{\prime}$ leads to a contradiction.

To state the second lemma of this appendix, we introduce some further notation. For $j \in N_{\ell l}, N_{\ell} \in \mathcal{N}^{E}$, we define $\kappa_{j}(g)=\kappa_{N_{\ell}}(g)=j^{\prime}$, where $j^{\prime}$ is the unique player in $N^{R}\left(N_{\ell} ; g\right)$ if this set is not empty. For any player $i \in N$, we define the set of links $\left\{\left(\kappa_{j}, i\right)\right\}$ to be empty if $N^{R}\left(N_{\ell} ; g\right)=\emptyset$. For example, consider Figure 8. In that network player $5=\kappa_{1}=\kappa_{9}=$ $\kappa_{N_{1}}=\kappa_{N_{3}}$, however $\kappa_{N_{2}}, \kappa_{N_{4}}$ and $\kappa_{i}$ for all $i \in N \backslash\{1,9\}$ are not defined. Thus $\left\{\left(\kappa_{1}, 4\right)\right\}=\{(5,4)\}$, but $\left\{\left(\kappa_{2}, 4\right)\right\}=\emptyset$. By Lemma 11, connectedness of $g$ and the fact that all links in $g$ are stable, we know that $\kappa_{N_{\ell}}$ sponsors precisely one link to $N_{\ell}$ in all his best replies to $g$.

Now, for some link $\left(i, i^{\prime}\right) \in g$ with $i \in N_{\ell}$ and $i^{\prime} \in N_{\ell^{\prime}}, \ell^{\prime} \neq \ell$, let $j$ be a player in $A_{i i^{\prime}}^{E}(g)$, i.e. $j$ is a player in some entirely externally connected group $N_{k}$ that is observed by $i$ via his link to $i^{\prime}$ and there is at least one other player in $N_{k}$ not observed via this link. When $\kappa_{j}$ exists, let $\kappa_{j}$ update his strategy by replacing his link to $j$ by a link to a player $j^{\prime}$ in $N_{k} \cap D_{i i^{\prime}}^{E}(g)$, thus to a player in $N_{k}$ not observed by $i$ through his link to $i^{\prime}$. Clearly, $\kappa_{j}$ plays a best reply, so also the new network belongs to $X$. However, $A_{i i^{\prime}}$ has be decreased, since all players that $j$ and thus also $i$ observed via the link $\left(\kappa_{j}, j\right)$ are not observed by $i$ though $\left(i, i^{\prime}\right)$ in the new network. Now, set $g_{0}=g$ and define iteratively for $h=1,2, \ldots$,

$$
g_{h}=\left(g_{h-1} \backslash\left(\cup_{j \in A_{i i^{\prime}}^{E}\left(g_{h-1}\right)}\left\{\left(\kappa_{j}, j\right)\right\}\right)\right) \cup\left(\cup_{j \in A_{i i^{\prime}}^{E}\left(g_{h-1}\right)}\left\{\left(\kappa_{j}, j^{\prime}\right)\right\}\right),
$$

where for each $j \in A_{i i^{\prime}}^{E}(g)$ it holds that $j^{\prime}$ is an arbitrarily chosen player in $N_{k} \cap D_{i i^{\prime}}^{E}(g)$ if $j \in N_{k}$. Within a finite number of steps this iterative procedure leads to a network $g_{h}$, say $g_{*}$, such that $A_{i i^{\prime}}^{E}\left(g_{*}\right)=\emptyset$ or $N^{R}\left(j ; g_{*}\right)=$ $\emptyset$ for any $j \in A_{i i^{\prime}}^{E}\left(g_{*}\right)$, so that $g^{h^{\prime}}=g_{*}=g^{h}$ for all $h^{\prime}>h$. Denoting $A_{i i^{\prime}}^{\min }(g)=A_{i i^{\prime}}\left(g_{*}\right)$, by construction we have that

$$
A_{i i^{\prime}}^{\min }(g) \subseteq A_{i i^{\prime}}\left(g_{h}\right) \text {, for all } h .
$$

Analogously, for some link $\left(i, i^{\prime}\right) \in g$ with $i \in N_{\ell}$ and $i^{\prime} \in N_{\ell^{\prime}}$, ell $\neq=\ell$, let $j$ be a player in $D_{i i^{\prime}}^{E}(g)$, i.e. $j$ is a player in some entirely externally connected group $N_{k}$ that is not observed by $i$ via his link to $i^{\prime}$ and there is at least one other player in $N_{k}$ that is observed via this link. When $\kappa_{j}$ exists, let $\kappa_{j}$ update his strategy by replacing his link to $j$ by a link to a player $j^{\prime}$ in $N_{k} \cap A_{i i^{\prime}}^{E}(g)$, thus to a player in $N_{k}$ observed by $i$ through his link to $i^{\prime}$. Again $\kappa_{j}$ plays a best reply and this also the new network belongs to $X$. However, now $A_{i i^{\prime}}$ increases with all players that $j^{\prime}$ now observes via the link $\left(\kappa_{j}, j^{\prime}\right)$. Setting $g^{0}=g$ and proceeding iteratively we obtain $h=1,2, \ldots$ the sequence of networks

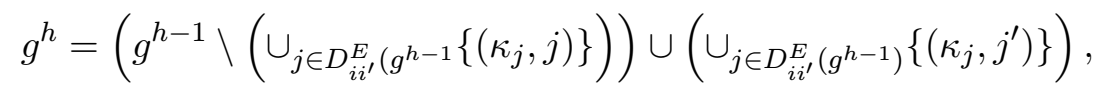

where for each $j \in D_{i i^{\prime}}^{E}(g)$ it holds that $j^{\prime}$ is an arbitrarily chosen player in $N_{k} \cap A_{i i^{\prime}}^{E}(g)$ if $j \in N_{k}$. Again, within a finite number of steps this 
iterative procedure leads to a network $g^{h}$, say $g^{*}$, such that $D_{i i^{\prime}}^{E}\left(g^{*}\right)=\emptyset$ or $N^{R}\left(j ; g^{*}\right)=\emptyset$ for any $j \in D_{i i^{\prime}}^{E}\left(g^{*}\right)$, so that $g^{h^{\prime}}=g^{*}=g^{h}$ for all $h^{\prime}>h$. Denoting $A_{i i^{\prime}}^{\max }(g)=A_{i i^{\prime}}\left(g^{*}\right)$, by construction we have that

$$
A_{i i^{\prime}}\left(g^{h}\right) \subseteq A_{i i^{\prime}}^{\max }\left(g^{*}\right) \text {, for all } h .
$$

We now have the following lemma.

Lemma 18. Let $X \subseteq \mathcal{G}$ be an MCS and let $i$ and $i^{\prime}$ be two players in different groups $N_{\ell}$ and $N_{\ell^{\prime}}$. Then for any two networks $g$ and $f$ in $X$ containing the link $\left(i, i^{\prime}\right)$ it holds that $\left.A_{i i^{\prime}}^{\min }(g)\right)=A_{i i^{\prime}}^{\min }(f)$ and $\left.A_{i i^{\prime}}^{\max }(g)\right)=$ $A_{i i^{\prime}}^{\max }(f)$.

Proof. Let us suppose for sake of contradiction that $A_{i i^{\prime}}^{\min }(g) \neq A_{i i^{\prime}}^{\min }(f)$, where $\left(i, i^{\prime}\right) \in g \cap f$. This implies that there is a player, say $j$, that is a member of one of these two sets, but not of both. W.l.o.g. we suppose that $j \in A_{i i^{\prime}}\left(g^{\prime}\right)=A_{i i^{\prime}}^{\min }(g)$ and $j \notin A_{i i^{\prime}}\left(f^{\prime}\right)=A_{i i^{\prime}}^{\min }(f)$. Thus there must exist a sequence of best replies starting from $g^{\prime}$ such that a player leaves the set $A_{i i^{\prime}}^{\min }$. However, by the construction of $A_{i i^{\prime}}^{\min }(g)$, all players that $j$ wants to link to are within the set $A_{i i^{\prime}}^{\min }(g)$. Hence, player $j$ cannot leave the set unless another player, say $j^{\prime} \in A_{i i^{\prime}}^{\min }(g)$, leaves this set first. This is true for all $j \in A_{i i^{\prime}}^{\min }(g)$. Thus any arbitrary $j \in A_{i i^{\prime}}^{\min }(g)$ can only leave the set $A_{i i^{\prime}}^{\min }(g)$ if another player leaves it first or simultaneously. Clearly, the former is impossible, whereas leaving simultaneously requires that the leaving players form a cycle, for they all sponsor a link to one of the other leaving players. Therefore the resulting network is not minimal and, by property A, not part of an MCS. Therefore this also forms a contradiction. Hence, if $j \in A_{i i^{\prime}}^{\min }(g)$ then $j \in A_{i i^{\prime}}^{\min }(f) \forall j \in A_{i i^{\prime}}^{\min }(g)$ and $\forall g, f \in\left\{\hat{g} \in X \mid\left(i, i^{\prime}\right) \in \hat{g}\right\}$, which contradicts $A_{i i^{\prime}}^{\min }(g) \neq A_{i i^{\prime}}^{\min }(f)$. A similar argument shows that $A_{i i^{\prime}}^{\max }(g)=A_{i i^{\prime}}^{\max }(f)$. Otherwise, w.l.o.g. there would exist $j \notin A_{i i^{\prime}}^{\max }(g), j \in A_{i i^{\prime}}^{\max }(f)$. But no player can enter $A_{i i^{\prime}}^{\max }(g)$ without another player entering that set first. Therefore it must be the case that $A_{i i^{\prime}}^{h}(g)=A_{i i^{\prime}}^{h}\left(g^{\prime}\right)$ for all $g, f \in\left\{\hat{g} \in X \mid\left(i, i^{\prime}\right) \in \hat{g}\right\}$, where $h \in\{\min , \max \}$.

The proof of Lemma 16 follows now immediately.

Proof Lemma 16. Since by construction $A_{i i^{\prime}}\left(g_{*}\right) \subseteq A_{i i^{\prime}}\left(g_{h}\right)$ for all $h=1,2, \ldots$, and $A_{i i^{\prime}}\left(g_{*}\right)=A_{i i^{\prime}}\left(f_{*}\right)$ for any $f, g \in X$ containing $\left(i, i^{\prime}\right)$, it follows that for any $g \in X$ with $\left(i, i^{\prime}\right) \in g$ holds that

$$
A_{i i^{\prime}}\left(g_{*}\right)=\cup_{\left\{g^{\prime} \in X \mid\left(i, i^{\prime}\right) \in g^{\prime}\right\}} A_{i i^{\prime}}\left(g^{\prime}\right)
$$

Hence for any $g$ containing $\left(i, i^{\prime}\right)$ it holds that

$$
A_{i i^{\prime}}^{\min }(X)=A_{i i^{\prime}}\left(g_{*}\right) \text {. }
$$

Analogously we have for any $g \in X$ containing $\left(i, i^{\prime}\right)$ that

$$
A_{i i^{\prime}}^{\max }(X)=A_{i i^{\prime}}\left(g^{*}\right) \text {. }
$$

